\title{
On the waiting time in quantum repeaters with probabilistic entanglement swapping
}

\author{
E. Shchukin ${ }^{*}$ F. Schmidt † $^{\dagger}$ and P. van Loock \\ Johannes-Gutenberg University of Mainz, Institute of Physics, Staudingerweg 7, 55128 Mainz
}

\begin{abstract}
The standard approach to realize a quantum repeater relies upon probabilistic but heralded entangled state manipulations and the storage of quantum states while waiting for successful events. In the literature on this class of repeaters, calculating repeater rates has typically depended on approximations assuming sufficiently small probabilities. Here we propose an exact and systematic approach including an algorithm based on Markov chain theory to compute the average waiting time (and hence the transmission rates) of quantum repeaters with arbitrary numbers of links. For up to four repeater segments, we explicitly give the exact rate formulae for arbitrary entanglement swapping probabilities. Starting with three segments, we explore schemes with arbitrary (not only doubling) and dynamical (not only predetermined) connections. The effect of finite memory times is also considered and the relative influence of the classical communication (of heralded signals) is shown to grow significantly for larger probabilities. Conversely, we demonstrate that for small swapping probabilities the statistical behavior of the waiting time in a quantum repeater cannot be characterized by its average value alone and additional statistical quantifiers are needed. For large repeater systems, we propose a recursive approach based on exactly but still efficiently computable waiting times of sufficiently small sub-repeaters. This approach leads to better lower bounds on repeater rates compared to existing schemes.
\end{abstract}

PACS numbers: 03.67.Mn, 03.65.Ud, 42.50.Dv

\section{INTRODUCTION}

Quantum repeaters are essential ingredients for largescale, fiber-based quantum networks because of the exponential decay of photonic quantum information along the optical communication channels. Besides recent alloptical approaches relying on experimentally still demanding quantum error correction procedures, memorybased quantum repeaters remain good candidates to realize long-range quantum communication [1]. In such quantum repeaters, probabilistic events like, especially, the heralded distribution of entangled Bell states over sufficiently small elementary channel segments become independent through the use of sufficiently long-lasting quantum memories, thus circumventing the exponential scaling when the segments are connected. Remarkably, the precise average waiting time, and hence the communication rate in a non-deterministic quantum repeater is unknown and computing this time has been a longstanding problem [10]. An exact analytic solution only exists for the special case of deterministic entanglement swapping; otherwise any existing work on quantum repeaters relies upon approximations or numerical simulations. In our work, we present a systematic scheme, based on a Markov formalism 11] and linear equation solving, that enables one to obtain the exact waiting times in practically relevant regimes. For a repeater as large as 1024 segments, we demonstrate that the trade-off between the computational efficiency and the prediction accuracy can be dealt with in a modular approach by re-

\footnotetext{
* evgeny.shchukin@gmail.com

$\dagger$ fschmi@students.uni-mainz.de

$\ddagger$ loock@uni-mainz.de
}

cursively applying exactly but still efficiently computable sub-chains like 32 times 32 segments. This provides increasingly more accurate but still computable lower bounds on the repeater rates. Furthermore, our method allows one to easily compute the full probability distribution of the random waiting time for non-deterministic quantum repeaters including those with arbitrary (not only doubling) and dynamical (not only predetermined) connections and including classical communication and finite memory effects.

Quantum repeaters enable one, in principle, to extend (optical-fiber-based) quantum communication schemes such as quantum cryptography to distances as large as $1000 \mathrm{~km}$ and beyond despite channel losses that typically increase exponentially with distance. Thanks to some recent results on the bounds for point-to-point communication [12, 13, there are now well-defined benchmarks for long-distance quantum communication. These bounds can be explored in terms of a secret key rate per mode (per channel use) and, when ignoring all imperfections besides channel losses, this corresponds to an optimal raw qubit transmission rate without intermediate stations like in a quantum repeater [14].

Since the standard approach to quantum repeaters is based on quantum memories and on (at least partially) probabilistic operations on entangled states (distribution, swapping, and purification) 15, some recent proposals consider small-scale versions of quantum repeaters with a minimal number of memory stations (repeater links) [16-19]. While the rate analysis for the smallest repeater with only two segments and one link is fairly straightforward, quantum repeaters with two or more links become increasingly complex to analyze when the entanglement swapping is probabilistic. Indeed there is no explicit expression for the average repeater waiting time in the lit- 
erature for such advanced cases [20]. However, entanglement swapping based on heralded but non-deterministic Bell measurements is a rather natural situation, especially in one of the most prominent approaches based on atomic ensembles and linear optics 21] where normally even the ideal (photonic) Bell measurement cannot exceed an efficiency of $1 / 2$ [22]. Thus, so far, the typical approximate rate formulas that have been applied depended on the assumption of sufficiently small probabilities [10, 23 25]. Nonetheless, the most efficient memory-based quantum repeater schemes would rely on high swapping probabilities, for instance, based on suitable atom-light interactions [26] or enhanced linear-optics Bell measurements 27-29. While exact analytical rate formulas are known only for the extreme case of fully deterministic entanglement swapping [30], in the present work, we will address the entire range of arbitrary swapping probabilities including a full statistical analysis beyond only average values. For large-scale repeaters, we propose to divide the whole system into smaller subrepeater chains, still sufficiently big to maintain the great accuracy of our formalism and sufficiently small to be efficiently computable. Errors that occur during the long-distance entanglement distribution in a quantum repeater can be included into the Markov-chain formalism. Here the focus will be on the distribution times, which, for example, in the context of quantum key distribution would be related to a quantum repeater raw rate [31.

The paper is structured as follows. In Sec. II we introduce the general setting and review existing results on the rate analysis in quantum repeaters. Sec. III then discusses some commonly used approximations and Sec. IV gives a detailed introduction into the Markov-chain formalism for quantum repeaters. Explicit examples of small repeaters and some special cases are presented in Sec. V. How to incorporate the effects of finite memory and classical communication times into our rate analysis is described in Secs. VII and VIII, respectively. An alternative, complementary approach based on generating functions is introduced in Sec. IX and a possible way to compress the Markov chain describing a quantum repeater system in order to make the analysis more efficient is discussed in Sec. X on lumpability. Finally, Sec. XI briefly describes a numerical validation of some of our analytical results, Sec. XII treats larger repeater systems based on a recursive application of our exact rate formulas, and Sec. XIII concludes the paper.

\section{GENERAL SETTING AND KNOWN RESULTS}

Let us consider a quantum repeater with $n$ identical segments of length $L_{0}$ and $n-1$ swappings between them. The initial entanglement distribution success probability is denoted by $p$ and the swapping success probability by $a$. The former includes the local state preparation efficiency and the channel transmission probabil-
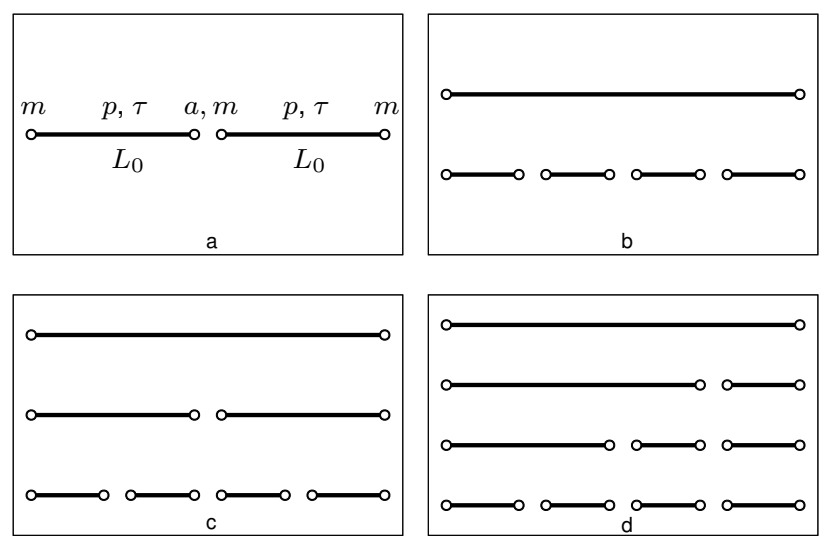

FIG. 1: Different ways for entanglement distribution in a quantum repeater. Fig. a illustrates the basic parameters of our model (see main text). Figs. b-d describe various distinct situations for the entanglement swapping (also see main text).

ity that decays exponentially with distance. The latter may include local losses such as (heralded) memory erasures. The characteristic time unit $\tau=L_{0} / \mathrm{c}$ is the typical duration of a single distribution attempt (including the transmission times of quantum and classical signals between neighboring repeater stations, and more specifically depending on the particular repeater protocol). To distribute entanglement along the whole repeater we first distribute it in individual segments and then combine them with swapping. If a swapping fails, or if a state is kept in memory longer than $\sim m$ time units $\tau$, the affected segments are zeroed out and have to start entanglement distribution from the very beginning. In Fig. 1 1 a we show two segments of such a quantum repeater. Figs. 1p-1k illustrate schemes based on deterministic and probabilistic entanglement swapping, respectively, while the latter has the fixed rule of doubling the distance on every nesting level. Fig. 1 $1 \mathrm{~d}$ is more general, no longer imposing such rules.

Generally, with probability $a^{n-1} p^{n}$ we succeed in one step with no gain over direct state transmission. In order to gain efficiency and change the scaling, we have to exploit quantum memories and allow to perform several steps to successfully distribute entanglement over the whole repeater. We introduce the random variable $K_{n}$ which is equal to the number of steps taken to successful distribution over the total distance $L=n L_{0}$ and, when multiplied with $\tau$, corresponds to the total waiting time (on higher nesting levels different time units may apply, see Sec. VIII). The goal of this work is to study statistical properties of $K_{n}$.

The main characteristic of any random variable is its probability distribution function (PDF). Up to now hardly anything has been known about the full PDF of $K_{n}$ in the most general setting with arbitrary (including still relevant) $p$ and $a$, although the waiting time in a sin- 
gle segment is obviously geometrically distributed with probability $p q^{k-1}$ for success at the $k$ th attempt (where $q=1-p$ ). A few results about the average $\bar{K}_{n}$ have been obtained. The expression for the average waiting time of a two-segment repeater with non-deterministic swapping and "memory cutoff" of $m$ time units has been obtained in Ref. [32] and reads as

$$
\bar{K}_{2}^{(m)}=\frac{1+2 q-2 q^{m+1}}{a p\left(1+q-2 q^{m+1}\right)} .
$$

In the case of deterministic swapping, $a=1$, and infinite memory, $m=+\infty$, the exact expression for $\bar{K}_{n}$ for arbitrary $n$ was presented in Ref. [30] (Fig. 10 illustrates such a repeater; since swappings cannot fail, there are no additional levels). It reads as

$$
\bar{K}_{n}=\sum_{j=1}^{n}(-1)^{j+1}\left(\begin{array}{l}
n \\
j
\end{array}\right) \frac{1}{1-q^{j}} .
$$

The expression for the average waiting time of an $n$ segment quantum repeater with deterministic swapping and a finite memory cutoff of $m$ time units has been obtained in 33 and it is given by the following expression:

$$
\bar{K}_{n}^{(m)}=\frac{1-\left(1-q^{m}\right)^{n}+\left(1-q^{n}\right)\left(m-\sum_{i=1}^{m-1}\left(1-q^{i}\right)^{n}\right)}{\left(1-q^{m+1}\right)^{n}-q^{n}\left(1-q^{m}\right)^{n}} .
$$

We thus have three expressions independently obtained by different methods, so we need to verify that they are in agreement where applicable. Simple algebra shows that the expression (2) for $n=2$ coincides with the expression (1) for $a=1$ and $m=+\infty$ and that the expression (1) for $a=1$ coincides with the expression (3) for $n=2$. Verification that the expression (3) for $m=+\infty$ coincides with the expression (2) requires slightly more work.

Lemma 1. The expressions (2) and (3) are consistent for the case of infinite memory cutoff: $\bar{K}_{n}^{(+\infty)}=\bar{K}_{n}$.

Proof. We need only verify the following relation:

$$
\lim _{m \rightarrow+\infty}\left(m-\sum_{j=1}^{m-1}\left(1-q^{j}\right)^{n}\right)=\bar{K}_{n} .
$$

The sum can be expanded as follows:

$$
\begin{aligned}
\sum_{i=1}^{m-1}\left(1-q^{i}\right)^{n} & =\sum_{j=0}^{n}(-1)^{j}\left(\begin{array}{c}
n \\
j
\end{array}\right) \sum_{i=1}^{m-1} q^{i j} \\
& =m-1+\sum_{j=1}^{n}(-1)^{j}\left(\begin{array}{c}
n \\
j
\end{array}\right) \frac{q^{j}-q^{j m}}{1-q^{j}} .
\end{aligned}
$$

We thus have

$$
\begin{aligned}
& \lim _{m \rightarrow+\infty}\left(m-\sum_{j=1}^{m-1}\left(1-q^{j}\right)^{n}\right) \\
& =1+\sum_{j=1}^{n}(-1)^{j+1}\left(\begin{array}{l}
n \\
j
\end{array}\right) \frac{q^{j}}{1-q^{j}}=\bar{K}_{n} .
\end{aligned}
$$

In the last equality we used the relations

$$
\frac{q^{j}}{1-q^{j}}=\frac{1}{1-q^{j}}-1
$$

and

$$
1-\sum_{j=1}^{n}(-1)^{j+1}\left(\begin{array}{l}
n \\
j
\end{array}\right)=(1-1)^{n}=0 .
$$

This concludes the proof that the expressions (2) and (3) are consistent.

One must be careful by using the expressions (2) and (3) for numerical evaluation of the averaged waiting time. For large $n$ (roughly speaking, for $n>60$ ) and for small $p$ (roughly, for $p<0.01$ ) one can obtain wrong results due to the limitations of the standard double precision floating point arithmetic. In this case, either one has to use multiple precision numbers or use the approximations presented in the next section.

\section{APPROXIMATIONS}

No expression for the exact average waiting time time is known in the case of non-deterministic swapping, $a<$ 1 , and for more than two segments, $n>2$. Moreover, for non-deterministic swapping different orders of the swapping operations lead to schemes of significantly different efficiencies. One of these schemes is when the number of segments is a power of two, $n=2^{d}$, recursively doubling the entangled segments in the repeater (see Fig. 1 this scheme, an often used approximation [10] is given by

$$
\bar{K}_{n} \approx\left(\frac{3}{2 a}\right)^{d} \frac{1}{p}=\left(\frac{3}{2 a}\right)^{\log _{2} n} \frac{1}{p} \equiv \bar{K}_{n}^{\prime} .
$$

As opposed to our exact treatment below, this approximation can be applied only for the power-of-two case and, as we will show, it is imprecise in relevant regimes of parameters $p$ and $a$. It is asymptotically precise only when both $p$ and $a$ are small. In particular, for small $p$ and bigger values of $a$ (a very common regime, see e.g. Ref. [34]) the approximation is much larger than the actual waiting times and hence the minimal repeater performance in terms of lower bounds on the repeater rates is significantly underestimated. Moreover, the approximation does not produce the correct scaling behavior. For the fully deterministic case $p=a=1$ we obviously have $\bar{K}_{n}=1$, because we immediately succeed in the very first attempt, but

$$
\bar{K}_{n}^{\prime}=(3 / 2)^{\log _{2} n}=n^{\log _{2} 3 / 2}=n^{0.58 \cdots}>\sqrt{n} .
$$

Thus, in this case the approximation is off by a factor of larger than $\sqrt{n}$. Neither the exact result $(2)$ nor the approximation (9) are directly applicable with a finite memory cutoff $m<+\infty$ or with inclusion of classical 
communication times at higher nesting levels. The exact result (3) for arbitrary $m$ and $a=1$ is based on basic probability theory, hard to systematically generalize. How to obtain a systematic and general framework and incorporate various effects such as arbitrary memory cutoffs and classical communication times with our formalism is described in detail in later sections of our work. Here we shall now focus on general repeaters for $m=+\infty$.

To find out the asymptotic behavior of $\overline{K_{n}}$ in the deterministic swapping case, $a=1$, and for $p \ll 1$, we expand $(1-p)^{j}$ with the Binomial Theorem and keep only the linear term in $p$ in each of the denominators in the sum (2). We have

$$
\bar{K}_{n} \approx \frac{1}{p} \sum_{j=1}^{n}(-1)^{j+1}\left(\begin{array}{l}
n \\
j
\end{array}\right) \frac{1}{j}=\frac{1}{p} \sum_{j=1}^{n} \frac{1}{j}=\frac{H_{n}}{p},
$$

where $H_{n}=\sum_{j=1}^{n} 1 / j$ is the $n$ 'th harmonic number. For large $n$ it can be approximated by

$$
H_{n}=\gamma+\ln (n)+\frac{1}{2 n}+\mathcal{O}\left(\frac{1}{n^{2}}\right)
$$

where $\gamma=0.57721 \ldots$ is the EulerMascheroni constant. Combining all these results, we get the following simple approximation:

$$
\bar{K}_{n} \approx \frac{1}{p}\left(\gamma+\ln (n)+\frac{1}{2 n}\right) .
$$

For a fixed number of segments $n$ both approximations, this and the commonly used $\bar{K}_{n}^{\prime}$, give the same asymptotic growth of the commonly time as $\mathcal{O}(1 / p)$, but $\bar{K}_{n}^{\prime}$ gives a wrong factor. For a fixed value of entanglement distribution success probability $p$, the waiting time really grows as $\mathcal{O}(\ln n)$, while $\bar{K}_{n}^{\prime}$ scales as $\mathcal{O}\left(n^{\log _{2}(3 / 2)}\right)=$ $\mathcal{O}\left(n^{0.58 \cdots}\right)$, which grows faster than $\mathcal{O}(\sqrt{n})$. This is a clear example where the usually employed approximations when used in the wrong regime, i.e., $p \ll 1$ and $a=1$ as still relevant for practical quantum repeaters, lead to an inaccurate scaling.

The approximation $\bar{K}_{n}^{\prime}$, given by Eq. (9), is applicable only in the power-of-two case, $n=2^{d}$, and for deterministic swapping this approximation becomes rather inaccurate. In the next section, we present an approach to compute the average waiting time exactly and for an arbitrary number of segments. This approach is based on the venerable Markov chain theory, which has many applications in different branches of mathematics, physics, biology and computer science.

\section{MARKOV CHAINS}

Roughly speaking, a Markov chain is a formal description of a system that can be in several states and can go from one state to another with known probability. We denote the state space of the system by $\mathcal{S}=\left\{s_{1}, \ldots, s_{N}\right\}$. The transition probability from the state $s_{i}$ to the state $s_{j}$ is expressed as $p_{i j}=\mathbf{P}\left(s_{i} \rightarrow s_{j}\right)$. The matrix $P=\left(p_{i j}\right)_{i, j=1}^{N}$ of these probabilities is referred to as the transition probability matrix (TPM) of the system. We can apply this formalism to study properties of the waiting time of quantum repeaters.

\section{A. Simple example}

To illustrate the description of quantum repeaters with the Markov chain approach, consider a two-segment repeater. It can be only in five states, which we denote as $00,01,10,11$ and $\overline{11}$, where 0 means that the segment has no distributed entanglement yet and 1 means that entanglement was successfully distributed. The overline means that the corresponding segments have been successfully swapped and represent now a single, longer segment. The transition probabilities between these states are easy to compute; the states with all possible transitions between them are shown in Fig. 2a. For example, the probabilities $\mathbf{P}(11 \rightarrow 00)=1-a$ and $\mathbf{P}(11 \rightarrow \overline{11})=a$ mean that from the state 11 , where both segments have successfully generated entanglement and are ready to be swapped, we can either succeed with probability $a$ and move to the state $\overline{11}$, or we fail with probability $1-a$ and move back to the initial state 00. The TPM of the two-segment repeater thus reads as

$$
P=\left(\begin{array}{ccccc}
q^{2} & p q & p q & p^{2} & 0 \\
0 & q & 0 & p & 0 \\
0 & 0 & q & p & 0 \\
1-a & 0 & 0 & 0 & a \\
0 & 0 & 0 & 0 & 1
\end{array}\right)
$$

The main property of any TPM is that all its row sums are equal to one, and this matrix obviously satisfies this property. Note that one state is special - there is no arrow originating from the state $\overline{11}$. Once the system entered this state, it will stay there. To be completely precise, we would need to show a loop for this state with probability 1 , but instead we distinguish it with double circle. Such states are referred to as absorbing.

A typical approach to study the waiting time is to ignore the time it takes to perform the swapping, as well as the classical communication time associated, in general, with any nesting level beyond the initial entanglement distribution (the inclusion of such more general effects will be discussed in Sec. VIII). Thus, we only count the initial entanglement distribution time (including its classical communication). The waiting time, the variable $K_{2}$ in the example, is thus the number of steps from the initial state 00 to the absorbing state $\overline{11}$ without counting the dashed zero-time transitions. This problem is closely related to the well-known problem for Markov chains: absorption time. The absorption time is the number of steps it takes to get from some starting state to an absorbing state (a general Markov chain can have 


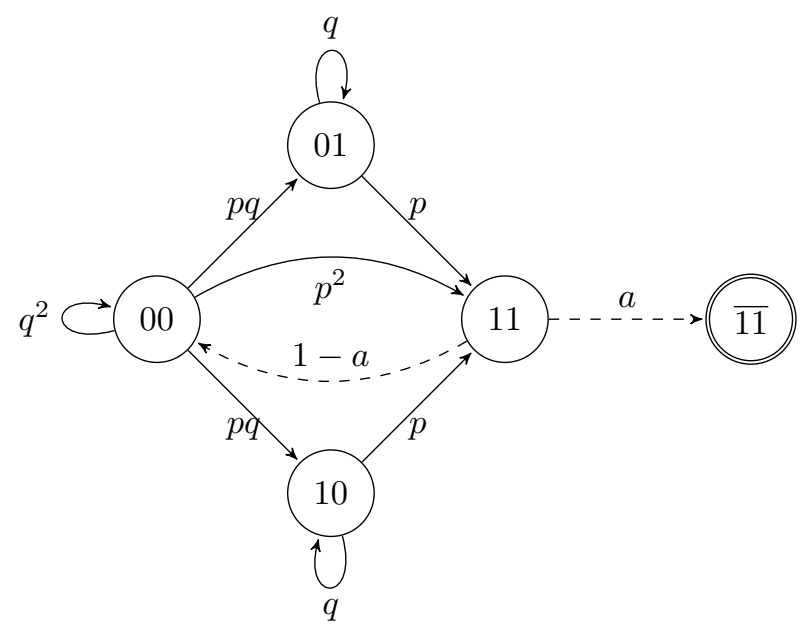

(a) With zero-time transitions.

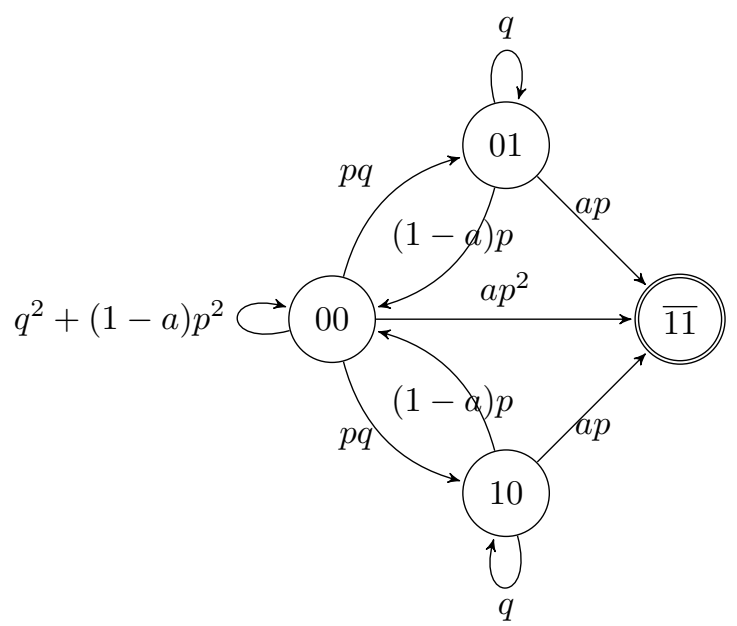

(b) Without zero-time transitions.

FIG. 2: Markov chain of a two-segment repeater.

more than one absorbing state). If we could describe the quantum repeater by a Markov chain without zero-time transitions, then the waiting time would be exactly the absorption time, whose properties can be obtained directly from the corresponding TPM. The Markov chain without zero-time transitions for a two-segment quantum repeater is shown in Fig. 2b. We just need to remove the state 11 and recompute the transition probabilities. We thus reduced the problem of studying the waiting time of this simplest repeater to the absorption time problem of the Markov chain shown in Fig. 2b with the following TPM:

$$
P_{2}=\left(\begin{array}{cccc}
q^{2}+(1-a) p^{2} & p q & p q & a p^{2} \\
(1-a) p & q & 0 & a p \\
(1-a) p & 0 & q & a p \\
0 & 0 & 0 & 1
\end{array}\right)
$$

This reduction illustrates a general phenomenon: either we use a larger chain with zero-time transitions, whose transition probabilities are easier to compute, or we use a more compact chain, but should spend more efforts to determine the transition probabilities. Below we show that this reduction to the absorption time problem is possible for a general $n$-segment quantum repeater, but before this we shall review the absorption time problem for general Markov chains.

\section{B. Absorption time}

Consider a Markov chain with a single absorbing state, which we assume to be the last one, $s_{N}$. The general theory can be applied to a chain with several absorbing states, but we need a simpler case when there is only one such state. In this case the TPM can be partitioned as follows:

$$
P=\left(\begin{array}{cc}
Q & \mathbf{u} \\
\mathbf{0}^{\mathrm{T}} & 1
\end{array}\right)
$$

where $Q$ is the matrix of transition probabilities between non-absorbing states, $\mathbf{u}$ is the vector of transition probabilities from non-absorbing states to the absorbing one and $\mathbf{0}$ is the zero vector. From the basic property of TPMs it is easy to see that $\mathbf{u}=(I-Q) \mathbf{1}$, where $I$ is the identity matrix of the appropriate dimension, $N-1$, and $\mathbf{1}=(1, \ldots, 1)^{T}$ is the vector of the same dimension with all components equal to 1 . For any state $s_{i}$ except the absorbing one $s_{N}$ we introduce a random variable $K(i)$ whose value is the number of steps it takes to get to the absorbing state from the state $s_{i}$ (for the absorbing state we would have $K(N)=0$, so it is not useful to introduce this variable). We combine all these variables into the vector

$$
\mathbf{K}=(K(1), \ldots, K(N-1))^{\mathrm{T}} .
$$

We now show that the full PDF of these random variables (and thus their averages, standard deviations and all higher moments) can be easily expressed in terms of the submatrix $Q$ of the TPM $P$.

Theorem 1. The probability distribution of $\mathbf{K}$ is given by the following simple expression:

$$
\mathbf{p}_{k}=Q^{k-1} \mathbf{u} .
$$

The average waiting time and the second moment read as

$$
\begin{aligned}
\overline{\mathbf{K}} & =R \mathbf{1}, \\
\overline{\mathbf{K}^{\circ 2}} & =(2 R-I) \overline{\mathbf{K}},
\end{aligned}
$$

where $\mathbf{x}^{\circ 2}$ is the component-wise square (also known as Hadamard square) of the vector $\mathbf{x}$ and $R=(I-Q)^{-1}$ is the fundamental matrix of the chain. The variance is $\boldsymbol{\sigma}^{2}=\overline{\mathbf{K}^{\circ 2}}-\overline{\mathbf{K}}^{\circ 2}$. 
Proof. For any non-absorbing state $s_{i}$ we have

$$
\left(\mathbf{p}_{k}\right)_{i}=\sum_{j_{1}, \ldots, j_{k-1}} Q_{i j_{1}} Q_{j_{1} j_{2}} \ldots Q_{j_{k-2} j_{k-1}} u_{j_{k-1}}
$$

where the summation is over all non-absorbing states since, if one of the intermediate states is the absorbing one, then we succeed in less then $k$ steps. In the vector form we have exactly Eq. (18). Now that we know the full PDF, we can compute the average waiting time and its standard deviation. By the definition of the average value and Eq. (18), we have

$$
\overline{\mathbf{K}}=\sum_{k=1}^{+\infty} k \mathbf{p}_{k}=\sum_{k=1}^{+\infty} k Q^{k-1} \mathbf{u} .
$$

It has been proven in Ref. 35] that the series $\sum_{k=1}^{+\infty} Q^{k-1}$ converges, so we have

$$
\begin{aligned}
\sum_{k=1}^{+\infty} k Q^{k-1} & =\left(\sum_{k=1}^{+\infty} Q^{k-1} t^{k}\right)_{t=1}^{\prime} \\
& =\left(t(I-Q t)^{-1}\right)_{t=1}^{\prime}=(I-Q)^{-2},
\end{aligned}
$$

where the prime denotes the derivative with regards to $t$. From this it immediately follows that

$$
\overline{\mathbf{K}}=(I-Q)^{-2} \mathbf{u}=(I-Q)^{-2}(I-Q) \mathbf{1}=R \mathbf{1} .
$$

The second moment can be computed in a similar way:

$$
\overline{\mathbf{K}^{\circ 2}}=\sum_{k=1}^{+\infty} k^{2} \mathbf{p}_{k}=\sum_{k=1}^{+\infty} k^{2} Q^{k-1} \mathbf{u} .
$$

We have

$$
\begin{aligned}
\sum_{k=1}^{+\infty} k^{2} Q^{k-1} & =\left(t\left(\sum_{k=1}^{+\infty} Q^{k-1} t^{k}\right)^{\prime}\right)_{t=1}^{\prime} \\
& =(I+Q)(I-Q)^{-3}
\end{aligned}
$$

so we get

$$
\overline{\mathbf{K}^{\circ 2}}=(I+Q)(I-Q)^{-2} \mathbf{1}=(I+Q)(I-Q)^{-1} \overline{\mathbf{K}} .
$$

We just need to show that $(I+Q)(I-Q)^{-1}=2(I-$ $Q)^{-1}-I$, which is trivially verified by multiplying both sides with $I-Q$. This concludes the proof.

This result shows that the fundamental matrix $R=$ $(I-Q)^{-1}$ is an important characteristic of the corresponding Markov chain. If we can obtain this inverse in a meaningful form analytically, we can immediately compute the desired characteristics of the waiting time. If it is infeasible to find the inverse analytically, we can go the numerical way. The vector of average waiting times $\overline{\mathbf{K}}$ is the solution of the following system of linear equations:

$$
(I-Q) \overline{\mathbf{K}}=\mathbf{1} .
$$

It is numerically more robust to solve the system 27) directly than to compute the matrix $R$ and multiply it by 1 . The second moment satisfies the system of linear equations

$$
(I-Q) \overline{\mathbf{K}^{\circ 2}}=(I+Q) \overline{\mathbf{K}} .
$$

Having found the first moment $\overline{\mathbf{K}}$, we can solve this system to obtain the second moment and the standard deviation. Similarly, higher moments can be obtained. We thus can compute all statistical properties of each random variable $K(i)$.

\section{Application to quantum repeaters}

Now we apply the general theory developed in the previous subsection to quantum repeaters and show that the problem of determining the statistical properties of the waiting time can be reduced to the absorption time problem of an appropriate Markov chain. In the previous subsection we demonstrated how to compute the absorption time of the system started in any state, but in the applications to quantum repeaters we usually need only the absorption time of the system started in the initial state (which we always assume to be the first), so we can just take the first component of the vectors $\overline{\mathbf{K}}$ and $\boldsymbol{\sigma}$. Unfortunately, we cannot solve a system of linear equations just for one variable without finding the values of the others, even if we later discard those other values.

Each segment of an $n$-segment quantum repeater can be in two states, either entanglement has been distributed (which happens with probability $p$ ) or it has not (with probability $q=1-p$ ). Moreover, some groups of segments can be successfully swapped, which we will denote by an overline over the swapped segments. The state of a quantum repeater can be fully described by $n$ digit binary strings with overlines over all possible groups of 1s; individual segments are shown without overlines. For a two-segment repeater there are five states, which were listed above. For a three-segment repeater, there are 13 states:

$$
\begin{array}{ccccccc}
000 & 010 & 100 & 110 & 0 \overline{11} & \overline{11} 0 & \overline{111} \\
001 & 011 & 101 & 111 & 1 \overline{11} & \overline{111} &
\end{array}
$$

The number of states grows exponentially with the number of segments. More precisely, the number of states is given by the following

Lemma 2. The number of states $N_{n}$ of an n-segment repeater is the $(2 n+1)$-th Fibonacci number: $N_{n}=F_{2 n+1}$.

Proof. The statement of the Lemma is correct for $n=2$ and $n=3$ : in the former case there are $F_{5}=5$ states, $00,01,10,11$ and $\overline{11}$, and in the latter case there are $F_{7}=13$ states as listed above. The odd-index Fibonacci numbers $F_{2 n+1}$ satisfy the following recurrence relation:

$$
F_{2 n+5}=3 F_{2 n+3}-F_{2 n+1},
$$


which is easy to obtain from the defining relation $F_{n+2}=$ $F_{n+1}+F_{n}$. We show that the numbers $N_{n}$ satisfy the relation

$$
N_{n+1}=3 N_{n}-N_{n-1},
$$

which will prove that $N_{n}=F_{2 n+1}$.

The set of all $(n+1)$-digit binary strings with overlines can be partitioned into two subsets $\mathcal{S}_{1}$ and $\mathcal{S}_{2}$ : the first subset contains those strings that do not end in an overline, and the second subset contains those strings that do. Every string from $\mathcal{S}_{1}$ can be obtained by suffixing all possible states of an $n$-segment repeater with zero and one, thus $\left|\mathcal{S}_{1}\right|=2 N_{n}$. It is easy to see that the strings of the set $\mathcal{S}_{2}$ are in one-to-one correspondence with the states of an $n$-segment repeater that end with one: the ending overline in an $n$-digit string $* \ldots * 1$ can be extended to $* \ldots \overline{* 11}$ or a new overline can be introduced if the last 1 is not overlined, $* \ldots * 1 \rightarrow * \ldots * \overline{11}$. It follows that $\left|\mathcal{S}_{2}\right|$ is the number of $n$-digit strings with overlines that end with one. This number can be obtained by subtracting from the total number of strings $N_{n}$ the number of strings that end with zero, which are in one-to-one correspondence with $(n-1)$-digit strings with overlines. We thus have the relation $\left|\mathcal{S}_{2}\right|=N_{n}-N_{n-1}$, which gives $N_{n+1}=\left|\mathcal{S}_{1}\right|+\left|\mathcal{S}_{2}\right|=3 N_{n}-N_{n-1}$, so the proof is complete.

From this Lemma it follows that the number of states grows exponentially as $N_{n}=F_{2 n+1}=\mathcal{O}\left(\lambda^{n}\right)$, with $\lambda=$ $\varphi^{2}=2.61 \ldots$, where $\varphi=(1+\sqrt{5}) / 2$ is the golden ratio constant. However, not all of them are really needed to describe the process of entanglement distribution. The actual number of states necessary to describe this process depends on the scheme used to perform swappings. We always start in the initial state $0 \ldots 0$, and not every state is reachable for a given swapping scheme. For example, for the doubling scheme, which is exclusively studied in the literature, in the case of $n=4$ the state $0 \overline{110}$ is unreachable, since such a swapping is forbidden in this scheme. On the other hand, for a scheme that we refer to as dynamical, when we swap everything that is ready, $0 \overline{110}$ is reachable, so both states 0110 and $0 \overline{110}$ must be included. Any scheme with a predetermined rule for the swappings, such as doubling, we refer to as fixed. Binary trees corresponding to fixed schemes for small quantum repeaters are shown in Fig. 3 .

Moreover, if we want to reduce the problem of waiting time computing to the absorption time problem, we also need to remove all zero-time transitions and recompute the transition probabilities between the remaining states. This will reduce the size of the corresponding Markov chain, but will also make the computation of transition probabilities slightly more complicated by comparison with the Markov chain with zero-time transitions. Below we show that for the doubling scheme, which is applicable only if the number of segments is a power of two, $n=2^{d}$, the number of states needed to implement the scheme without zero-time transitions is $N_{n}^{\text {rec }}=2^{n}=2^{2^{d}}$.

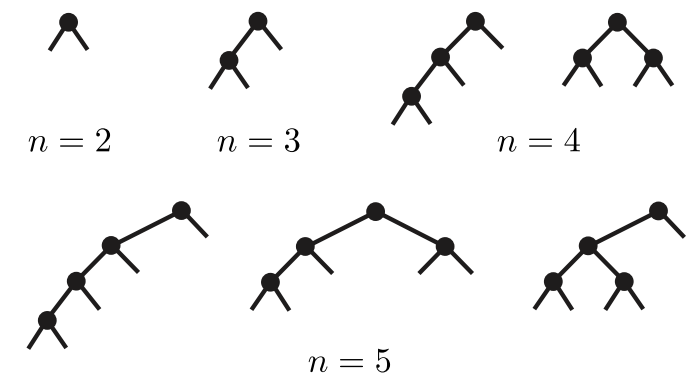

FIG. 3: Fixed schemes for small quantum repeaters.

In this "recursive" scheme we divide the segments into two equal parts, wait when both are ready and then try to perform the last swapping to distribute the entanglement over the whole repeater. We apply the same procedure to each half recursively, which is always possible since the number of segments is a power of two. This is illustrated by Fig. 11. Different schemes may require different subsets of the full set of states, but because the doubling scheme is the only one that has been analyzed in the literature, we mainly use this scheme to illustrate our method. Nonetheless, the method is completely general and can be applied to any swapping scheme. One of such more general (non-doubling) schemes is illustrated by Fig. 11.

We illustrate the process of removing zero-state transitions and the recomputation of transition probabilities by the case of $n=3$ and a fixed scheme when we try to swap segments 1 and 2 first and then, having successfully swapped them, try to swap the combined segments with segment 3 . Since we ignore the time it takes to perform swapping and the classical communication time needed to restart the process in the case of swapping failure, transitions between these states take different times. For example, the transition $000 \rightarrow 000$ takes one time unit (and happens with probability $q^{3}$ ), while the transition $011 \rightarrow 000$ happens instantaneously (with probability $1-a$ ). The states needed to implement this scheme without zero-time transitions are

$$
\begin{array}{llllllll}
000 & 001 & 010 & 011 & 100 & 101 & \overline{110} & \overline{111} .
\end{array}
$$

For example, the state $0 \overline{11}$ is unreachable from the initial state 000 and should not be included into this set. The state 110 has a zero-time transition to the state $\overline{11} 0$ and should not be included as well. Note that the set of states depends on the chosen scheme. If we consider a scheme where we swap segments 2 and 3 first and then swap them with segment 1 , the set of states reads as

$$
\begin{array}{llllllll}
000 & 100 & 001 & 101 & 010 & 110 & 011 & \overline{111} .
\end{array}
$$

Below we show how to determine this set of states for any fixed scheme with an arbitrary number of segments $n$, but now we illustrate how to recompute the probabilities. Consider the transition $000 \rightarrow 000$. In the original Markov chain with zero-time transitions the probability of this transition is $\mathbf{P}(000 \rightarrow 000)=q^{3}$. In the 
new, smaller chain, we can stay in the state 000 in three ways: (i) none of the entanglement distributions in the three segments succeed; this happens with probability $q^{3}$, (ii) the segments 1 and 2 succeed, but swapping them fails; this happens with probability $(1-a) p^{2} q$, (iii) all three segments succeed, swapping the first two also succeeds, but swapping them with the last one fails; this happens with probability $a(1-a) p^{3}$. We thus see that the new transition probability is the sum of these terms, $\mathbf{P}(000 \rightarrow 000)=q^{3}+(1-a) p^{2} q+a(1-a) p^{3}$. This example illustrates what we meant by saying that the new Markov chain will be smaller, but the transition probabilities will be more tricky to compute. In the next subsection we present an algorithm to compute the TPM of the Markov chain corresponding to any fixed scheme. Our approach is applicable to arbitrary schemes, not only to fixed ones, but the TPMs of dynamical schemes must be constructed by other means. Ones the TPM is constructed, we can use Eqs. 18)-19 to compute the statistical characteristics of the chosen scheme.

\section{Algorithm for TPM construction}

We now describe the algorithm for constructing the TPM $P$ of a quantum repeater constructed from two subrepeaters. We show how to express its TPM $P$ in terms of the sub-repeater TPMs $P^{\prime}$ and $P^{\prime \prime}$. The schemes used for these smaller repeaters can be arbitrary. They could also be fixed (and thus $P^{\prime}$ and $P^{\prime \prime}$ could be obtained by recursive applications of this algorithm), or they could be dynamical, or one fixed and the other dynamical any possible combination will work. When both smaller repeaters successfully distribute entanglement over them, we try to perform the last swapping and either succeed and distribute entanglement over the whole repeater or fail and have to start this process from scratch. If the schemes used for smaller repeaters are also fixed all the way down, then the whole scheme is fixed - the order we perform swappings is fixed and does not depend on the order in which the segments successfully distribute entanglement. But, as we have already noted, these schemes do not have to be fixed. The only restriction of this construction is that the division of the segments is fixed on the highest level, and what happens below can be arbitrary.

Let us assume that an $n$-segment repeater is divided into two parts with $n^{\prime}$ and $n^{\prime \prime}$ segments, so that $n=n^{\prime}+$ $n^{\prime \prime}$. The schemes of the smaller repeaters are described by the states $s_{i}^{\prime}, i=1, \ldots, N^{\prime}$, and $s_{j}^{\prime \prime}, j=1, \ldots, N^{\prime \prime}$, respectively. As usual, we assume that $s_{1}^{\prime}=0 \ldots 0\left(n^{\prime}\right.$ zeros) and $s_{1}^{\prime \prime}=0 \ldots 0$ ( $n^{\prime \prime}$ zeros $)$ are the initial states of the sub-repeaters and $s_{N^{\prime}}^{\prime}=\overline{1 \ldots 1}, s_{N^{\prime \prime}}^{\prime \prime}=\overline{1 \ldots 1}$ are the absorbing ones. Because swapping between the segments of different sub-repeaters are forbidden, the scheme for the whole repeater can be described by the states $s_{i}^{\prime} s_{j}^{\prime \prime}$ (which means the concatenation of strings) with one exception: the state $s_{N^{\prime}}^{\prime} s_{N^{\prime \prime}}^{\prime \prime}=\overline{1 \ldots 1} \overline{1 \ldots 1}$ must be replaced by the absorbing state $\overline{1 \ldots 1}$ ( $n$ ones) for the whole repeater. We thus have that this schemes requires $N=N^{\prime} N^{\prime \prime}$ states.

To illustrate this construction, consider a 3-segment repeater. We can divide it in only two ways: either like $--\mid-$ or like $-\mid--$. The states of a 1-segment "repeater" are 0,1 , and the states of a 2 -segment repeater (without zero-time transitions) are $00,01,10, \overline{11}$. In the first case $s_{i}^{\prime}$ denote the 1 -segment repeater states and $s_{j}^{\prime \prime}$ denote the 2-segment repeater states. It is easy to see that for such a division we obtain exactly the states (31) for the whole repeater. For the second division we get the states 32 .

We now need to compute transition probabilities between different states $s_{i}^{\prime} s_{j}^{\prime \prime}$. As a general rule, we have

$$
\mathbf{P}\left(s_{i}^{\prime} s_{j}^{\prime \prime} \rightarrow s_{k}^{\prime} s_{l}^{\prime \prime}\right)=\mathbf{P}^{\prime}\left(s_{i}^{\prime} \rightarrow s_{k}^{\prime}\right) \mathbf{P}^{\prime \prime}\left(s_{j}^{\prime \prime} \rightarrow s_{l}^{\prime \prime}\right),
$$

provided that $s_{k}^{\prime} s_{l}^{\prime \prime}$ is neither the initial nor the absorbing state of the whole repeater. For the absorbing state we have the following simple rule:

$$
\begin{aligned}
\mathbf{P}\left(s_{i}^{\prime} s_{j}^{\prime \prime}\right. & \rightarrow \overline{1 \ldots 1}) \\
& =a \mathbf{P}^{\prime}\left(s_{i}^{\prime} \rightarrow \overline{1 \ldots 1}\right) \mathbf{P}^{\prime \prime}\left(s_{j}^{\prime \prime} \rightarrow \overline{1 \ldots 1}\right),
\end{aligned}
$$

because the overall success is determined by the success of the transitions inside the sub-repeaters and the success of the swapping at the outermost level. For the initial state the rule is slightly more complicated and reads as

$$
\begin{aligned}
\mathbf{P}\left(s_{i}^{\prime} s_{j}^{\prime \prime}\right. & \rightarrow 0 \ldots 0) \\
& =\mathbf{P}^{\prime}\left(s_{i}^{\prime} \rightarrow 0 \ldots 0\right) \mathbf{P}^{\prime \prime}\left(s_{j}^{\prime \prime} \rightarrow 0 \ldots 0\right) \\
& +(1-a) \mathbf{P}^{\prime}\left(s_{i}^{\prime} \rightarrow \overline{1 \ldots 1}\right) \mathbf{P}^{\prime \prime}\left(s_{j}^{\prime \prime} \rightarrow \overline{1 \ldots 1}\right),
\end{aligned}
$$

since we can return to the initial state in two ways inside each sub-repeater individually or by going to the top and failing to swap on the outermost level. All these relations, the general one given by Eq. (33) and the two exceptions, given by Eqs. (34)-(35), can be written in a compact matrix form. In the case of $a=1$ we would have a very simple relation $P=P^{\prime} \otimes P^{\prime \prime}$, but in the nondeterministic case this relation must be modified. Namely, if we follow our standard convention that the initial state is the first in the TPM and the absorbing state is the last, then $P$ is obtained from $P^{\prime} \otimes P^{\prime \prime}$ by adding the last column, multiplied by $1-a$, to the first column, and then multiplying the last column by $a$ (the elements of the last row are untouched). In NumPy [36] notation this lengthy explanation can be compactly expressed as

$$
\begin{aligned}
& P=\operatorname{np} . \operatorname{kron}\left(P^{\prime}, P^{\prime \prime}\right) \\
& P[:-1,0]+=(1-a) * P[:-1,-1] \\
& P[:-1,-1] *=a
\end{aligned}
$$

This algorithm can be easily translated to other languages and computer algebra systems. We emphasize that in this algorithm the sub-repeaters may have different numbers of segments, but the division of the repeater 
into sub-repeaters is fixed - we know beforehand when we will try the outermost swapping. The schemes used for the sub-repeaters are arbitrary, not necessarily fixed. If we use the fixed schemes for the sub-repeaters, then we can recursively construct the TPM of the whole repeater with this algorithm, which holds, for example, for the standard doubling scheme. For the doubling scheme we start with the matrix $P_{1}$ of size 2 of a single segment and double it $d$ times to get the TPM $P_{n}$ of an $n$-segment repeater, where $n=2^{d}$. Because doubling squares the size of the TPM, $P_{n}$ is of size $2^{2^{d}}=2^{n}$, as we claimed before.

\section{EXAMPLES}

We now demonstrate how to apply the algorithm of the previous section to small repeaters, where computing TPMs by hand is still feasible. Moreover, here we also discuss dynamical schemes and compare their performance with that of fixed schemes.

\section{A. One-segment repeater}

This is a completely trivial case, but we nevertheless include it to utilize it later as a building block of larger repeaters. For a one-segment repeater the swapping success probability is not applicable, so in this case we have only one parameter $p$. This repeater can be described by $F_{3}=2$ states 0 and 1 . The TPM of such a repeater reads as

$$
P_{1}=\left(\begin{array}{ll}
q & p \\
0 & 1
\end{array}\right)
$$

The $1 \times 1$ matrix $Q_{1}$ can be identified with a number $q$, so from the relations $(19)$ we get

$$
\bar{K}_{1}=(1-q)^{-1} 1=\frac{1}{p}, \quad \overline{K_{1}^{2}}=\frac{1+q}{1-q} \bar{K}_{1}=\frac{2-p}{p^{2}} .
$$

We thus have $\sigma_{1}^{2}=\overline{K_{1}^{2}}-\bar{K}_{1}^{2}=q / p^{2}$. From this relation we see that $\sigma_{1} / K_{1}=\sqrt{q}$ and for small $p$ this ratio is close to one. It means that for small $p$ the waiting time has a large deviation and cannot be precisely characterized by its average value alone. Of course, we could easily obtain all these results directly from the geometric distribution of a single repeater segment, but our method is applicable to larger repeaters too, where it gives the desired result much more easily than by computing the individual probabilities.

\section{B. Deterministic swapping}

Here we consider an $n$-segment repeater with deterministic swapping. Because swappings cannot fail, their order does not matter and all schemes are equivalent in this case. We need $2^{n}$ states to describe this scheme. We can identify these states with the subsets of the set $[n]=\{1, \ldots, n\}$ of the segments (written as binary strings, we should overline the consecutive runs of 1 ). For example, in the case of $n=3$ these states are

$$
\begin{array}{llllllll}
000 & 001 & 010 & 0 \overline{11} & 100 & 101 & \overline{11} 0 & \overline{111} .
\end{array}
$$

The transition probability between the states associated with the subsets $I, J \subseteq[n]$ is given by

$$
\mathbf{P}(I \rightarrow J)=\left\{\begin{array}{ll}
0 & I \nsubseteq J \\
p^{|J|-|I|} q^{n-|J|} & I \subseteq J
\end{array} .\right.
$$

Nonzero transition probabilities are only from a set to any of its supersets since the set of ready segments can only increase with each transition, but never decrease. We first need to check that such an assignment of probabilities is correct, i.e., that the equality

$$
\sum_{J \subseteq[n]} \mathbf{P}(I \rightarrow J)=1
$$

is satisfied for all $I \subseteq[n]$. We have

$$
\begin{aligned}
\sum_{J \subseteq[n]} \mathbf{P}(I \rightarrow J) & =\sum_{J \supseteq I} \mathbf{P}(I \rightarrow J) \\
& =\sum_{j=|I|}^{n}\left(\begin{array}{c}
n-|I| \\
j-|I|
\end{array}\right) p^{j-|I|} q^{n-j} \\
& =\sum_{j=0}^{n-|I|}\left(\begin{array}{c}
n-|I| \\
j
\end{array}\right) p^{j} q^{n-|I|-j}=1,
\end{aligned}
$$

since the last sum is just $(p+q)^{n-|I|}=1$ by definition of $q=1-p$. We thus have a correctly constructed Markov chain without zero-transitions describing the entanglement distribution process in a quantum repeater with deterministic swapping. To find the absorption time we need to solve the system of $2^{n}-1$ equations (27) for the vector $\overline{\mathbf{K}}=\{\bar{K}(I)\}$, where the components are labeled by the strict subsets $I \subset[n]$. We now show that the solution reads as

$$
\bar{K}(I)=\sum_{j=1}^{n-|I|}(-1)^{j+1}\left(\begin{array}{c}
n-|I| \\
j
\end{array}\right) \frac{1}{1-q^{j}} .
$$

This expression for the empty set $I=\varnothing$ gives exactly the expression (2). We need to check that

$$
\sum_{J \subseteq[n]}\left(\delta_{I J}-\mathbf{P}(I \rightarrow J)\right) \bar{K}(J)=1,
$$

which can be rewritten as

$$
1+\sum_{J \supseteq I} \mathbf{P}(I \rightarrow J) \bar{K}(J)=\bar{K}(I) .
$$


The full proof is simple, but tedious so we just give two hints. The first thing to note is that the sum over all supersets of $I$ can be replaced by the summation over a simple index $i$ from 0 to $n-|I|$, introducing an additional factor $\left(\begin{array}{c}n-|I| \\ i\end{array}\right)$ (there are so many supersets $J \supseteq I$ with $|J|=|I|+i)$. The other hint is that the double sum that appears after this transformation can be simplified with the following rule:

$$
\sum_{i=0}^{n-|I|} \sum_{j=1}^{n-|I|-i}=\sum_{j=1}^{n-|I|} \sum_{i=0}^{n-|I|-j}
$$

The rest of the proof is just juggling with Binomial coefficients and applying the Binomial Expansion Theorem. We have just reproduced the well-known result for the waiting time of a general repeater with deterministic swapping 30.

\section{Asymmetric case}

In this subsection, we generalize the result of the previous section and consider deterministic swapping with asymmetric distribution probabilities. We no longer assume that all segments have the same entanglement distribution probability $p$ : the $i$-th segment has its own probability $p_{i}$. We use the notation

$$
p_{I}=\prod_{i \in I} p_{i}, \quad q_{I}=\prod_{i \in I} q_{i}
$$

for the product of probabilities over a set $I \subseteq[n]$. Note that in the case of all probabilities being equal, $p_{i}=p$, we simply have $p_{I}=p^{|I|}$. We now derive an explicit expression for the average waiting time of such an asymmetric repeater with deterministic swapping.

The transition probabilities read as

$$
\mathbf{P}(I \rightarrow J)=\left\{\begin{array}{ll}
0 & I \nsubseteq J \\
p_{J \backslash I} q_{\bar{J}} & I \subseteq J
\end{array},\right.
$$

where $\bar{J}=[n] \backslash J$ is the complement of $J \subseteq[n]$. One can check that

$$
\begin{aligned}
& \sum_{J \subseteq[n]} \mathbf{P}(I \rightarrow J)=\sum_{J \supseteq I} \mathbf{P}(I \rightarrow J) \\
& =\sum_{J \supseteq I} p_{J \backslash I} q \bar{J}=\prod_{i \in \bar{I}}\left(p_{i}+q_{i}\right)=1,
\end{aligned}
$$

for all $I \subseteq[n]$, so these probabilities satisfy the Markov chain property. We now show that the explicit solution of the system (27) in this case reads as

$$
\bar{K}(I)=\sum_{\varnothing \subset J \subseteq \bar{I}} \frac{(-1)^{|J|+1}}{1-q_{J}} .
$$

We need to check that these functions satisfy the equalities (45). Substituting Eq. (50) into Eq. (45), we will get a double sum, which can be transformed as follows:

$$
\sum_{J \supseteq I} \sum_{\varnothing \subset J^{\prime} \subseteq \bar{J}}=\sum_{\varnothing \subset J^{\prime} \subseteq \bar{I}} \sum_{I \subseteq J \subseteq \overline{J^{\prime}}} .
$$

We have

$$
\sum_{I \subseteq J \subseteq \bar{J}^{\prime}} p_{J \backslash I} q_{\bar{J}}=q_{J^{\prime}} \prod_{i \in \overline{J^{\prime}} \backslash I}\left(p_{i}+q_{i}\right)=q_{J^{\prime}},
$$

so we get

$$
\begin{aligned}
1 & +\sum_{J \supseteq I} \mathbf{P}(I \rightarrow J) \bar{K}(J)=1+\sum_{\varnothing \subset J^{\prime} \subseteq \bar{I}} \frac{(-1)^{\left|J^{\prime}\right|+1}}{1-q_{J^{\prime}}} q_{J^{\prime}} \\
& =\sum_{J^{\prime} \subseteq \bar{I}}(-1)^{\left|J^{\prime}\right|}+\sum_{\varnothing \subset J^{\prime} \subseteq \bar{I}} \frac{(-1)^{\left|J^{\prime}\right|+1}}{1-q_{J^{\prime}}}=\bar{K}(I) .
\end{aligned}
$$

Note that if all $p_{i}=p$ are equal, the expression 50 reduces to Eq. 43 , since for each $j=1, \ldots, n-|I|$ there are $\left(\begin{array}{c}n-|I| \\ j\end{array}\right)$ sets $J$ such that $\varnothing \subset J \subseteq \bar{I}$ and all these sets correspond to the same $q_{J}=q^{|J|}=q^{j}$. In the following subsections we start analyzing the more general and subtle case of non-deterministic swapping, $a<1$, for small repeaters.

\section{Two-segment repeater}

In the case of a two-segment repeater both parameters $p$ and $a$ are meaningful. This case has been considered before, so here we just briefly discuss it. The TPM (without zero-time transitions) is given by Eq. [15). Note that this matrix can be obtained by applying the algorithm (36) to the matrix (37). The fundamental matrix $R_{2}=\left(\bar{I}-Q_{2}\right)^{-1}$ can be easily obtained and it becomes

$$
R_{2}=\frac{1}{a p(2-p)}\left(\begin{array}{ccc}
1 & q & q \\
1-a & a+q & (1-a) q \\
1-a & (1-a) q & a+q
\end{array}\right)
$$

For the vector $\overline{\mathbf{K}}=R_{2} \mathbf{1}$ of average values, we have

$$
\overline{\mathbf{K}}=\frac{1}{a p(2-p)}\left(\begin{array}{c}
1+2 q \\
1+2 q-a q \\
1+2 q-a q
\end{array}\right)
$$

It is easy to see that the first element of this vector, $\bar{K}_{2}$, coincides with the expression (1) for $m=+\infty$, so we have just reproduced the well-known result for a two-segment repeater with non-deterministic swapping. The variance now reads as

$$
\sigma_{2}^{2}=\bar{K}_{2}^{2}-\frac{2 p^{3}-3 p^{2}-2 p+4}{a p^{2}(2-p)^{2}} .
$$


For the ratio of the standard deviation to the average value in the typical case of small $p$, we have

$$
\lim _{p \rightarrow 0} \frac{\sigma_{2}}{\bar{K}_{2}}=\sqrt{1-\frac{4}{9} a} .
$$

For small $a$ we again have $\sigma_{2} / \bar{K}_{2} \approx 1$. In the more practical situation of large $a$ the standard deviation is smaller (relative to the average value), but it never becomes smaller than $(\sqrt{5} / 3) \bar{K}_{2} \approx 0.75 \bar{K}_{2}$. So, even in this case the random variable $K_{2}$ cannot be accurately characterized only by its average value.

\section{E. Three-segment repeater}

This is the first example of a not-power-of-two case. Here we have two fixed schemes (which are statistically equivalent and represented by the $n=3$-scheme in Fig. 3 and one dynamical scheme for performing swappings. The two fixed schemes, segments 1 and 2 first, then segment 3 and segments 2 and 3 first, then segment 1 are equivalent and have identical statistical properties. So, effectively, we have only one fixed scheme in this case. The TPM of this scheme can be obtained from the matrices $P^{\prime}=P_{1}$ and $P^{\prime \prime}=P_{2}$ according to the algorithm (36), corresponding to the second scheme (segments 2 and 3 first, then segment 1). The TPM $P_{3}$ and the corresponding $\bar{K}_{3}$ become

$$
\begin{aligned}
P_{3} & =\left(\begin{array}{cccccccc}
q^{3}+(1-a) p^{2} q+a(1-a) p^{3} & p q^{2} & p q^{2} & a p^{2} q & p q^{2}+(1-a) p^{3} & p^{2} q & p^{2} q & a^{2} p^{3} \\
(1-a) p q+a(1-a) p^{2} & q^{2} & 0 & a p q & (1-a) p^{2} & p q & 0 & a^{2} p^{2} \\
(1-a) p q+a(1-a) p^{2} & 0 & q^{2} & a p q & (1-a) p^{2} & 0 & p q & a^{2} p^{2} \\
(1-a) p & 0 & 0 & q & 0 & 0 & 0 & a p \\
a(1-a) p^{2} & 0 & 0 & 0 & q^{2}+(1-a) p^{2} & p q & p q & a^{2} p^{2} \\
a(1-a) p & 0 & 0 & 0 & (1-a) p & q & 0 & a^{2} p \\
a(1-a) p & 0 & 0 & 0 & (1-a) p & 0 & q & a^{2} p \\
0 & 0 & 0 & 0 & 0 & 0 & 0 & 1
\end{array}\right), \\
\bar{K}_{3} & =\frac{a^{2}\left(p^{4}-5 p^{3}+10 p^{2}-10 p+4\right)+a\left(2 p^{4}-9 p^{3}+17 p^{2}-16 p+6\right)-4 p^{3}+16 p^{2}-23 p+12}{a^{2} p(2-p)\left(a\left(-p^{3}+3 p^{2}-4 p+2\right)+2 p^{2}-5 p+4\right)} .
\end{aligned}
$$

This time the expression for the fundamental matrix is not so compact as in the 2-segment case, but this is not a problem for computer algebra systems. One can compute the standard deviation in the same way as we did it before, but here we present only the limit of the ratio for small $p$ :

$$
\lim _{p \rightarrow 0} \frac{\sigma_{3}}{\bar{K}_{3}}=\frac{\sqrt{48 a^{4}-208 a^{3}+68 a^{2}+144 a+144}}{4 a^{2}+6 a+12} .
$$

For small $a$ we again have $\sigma_{3} / \bar{K}_{3} \approx 1$, while for large $a$ this ratio $\approx 7 / 11 \approx 0.64$.

We do not have to always follow a fixed way of per- forming swappings and may also try to swap any ready segments. In this case we need a different subset of the full set of states to describe this scheme:

$\begin{array}{llllllll}000 & 001 & 010 & 0 \overline{11} & 100 & 101 & \overline{110} & \overline{111} .\end{array}$

Note that these coincide with the states 39 for the case of a 3-segment repeater with deterministic swapping. This is not surprising, since in the deterministic swapping case, all schemes are equivalent and thus can be described by the same set of states. The TPM $P_{3}^{(\text {dyn })}$ and the corresponding $\bar{K}_{3}^{(\mathrm{dyn})}$ read as 


$$
\begin{gathered}
P_{3}^{\text {(dyn) }}=\left(\begin{array}{cccccccc}
q^{3}+2(1-a) p^{2} q+a(1-a) p^{3} & p q^{2}+(1-a) p^{3} & p q^{2} & a p^{2} q & p q^{2} & p^{2} q & a p^{2} q & a^{2} p^{3} \\
(1-a) p q+a(1-a) p^{2} & q^{2}+(1-a) p^{2} & 0 & a p q & 0 & p q & 0 & a^{2} p^{2} \\
2(1-a) p q+a(1-a) p^{2} & (1-a) p^{2} & q^{2} & a p q & 0 & 0 & a p q & a^{2} p^{2} \\
(1-a) p & 0 & 0 & q & 0 & 0 & 0 & a p \\
(1-a) p q+a(1-a) p^{2} & (1-a) p^{2} & 0 & 0 & q^{2} & p q & a p q & a^{2} p^{2} \\
a(1-a) p & (1-a) p & 0 & 0 & 0 & q & 0 & a^{2} p \\
(1-a) p & 0 & 0 & 0 & 0 & 0 & q & a p \\
0 & 0 & 0 & 0 & 0 & 0 & 0 & 1
\end{array}\right), \\
\bar{K}_{3}^{(\mathrm{dyn})}=\frac{a^{2}\left(p^{4}-4 p^{3}+6 p^{2}-5 p+2\right)+a\left(2 p^{4}-10 p^{3}+21 p^{2}-22 p+9\right)-4 p^{3}+16 p^{2}-22 p+11}{a^{2} p(2-p)\left[a\left(-p^{3}+2 p^{2}-2 p+1\right)+3 p^{2}-7 p+5\right]} .
\end{gathered}
$$

In this case we cannot apply the algorithm (36), since this scheme is dynamical, and the TPM has been computed directly from the definition of transition probabilities. We highlight the computation of some matrix elements. For example, the transition $000 \rightarrow 000$ is possible in four ways: (i) no entanglement distribution is successful; probability is $q^{3}$, (ii) the distribution in segments 1 and 2 was successful, but the swapping failed; probability is $(1-a) p^{2} q$, (iii) the distribution in segments 2 and 3 was successful, but the swapping failed; probability is $(1-a) p^{2} q$, (iv) the distribution in all three segments was successful, one swapping was also successful, but the second swapping failed; probability is $a(1-a) p^{3}$. Summing the probabilities of all these exclusive possibilities, we obtain the corresponding element of the TPM. In the case of a tie, when all segments successfully distribute entanglement simultaneously, we always try to swap segments 1 and 2 first. Since the two possibilities are equivalent, it does not matter whether we do this or first try to swap segments 2 and 3. We may even randomly choose between these two options. The matrix is constructed under the assumption that segments 1 and 2 are always tried first, and that is why the probabilities of the transitions $001 \rightarrow 001$ and $100 \rightarrow 100$ (and some others) differ. We can stay in the state 001 in two ways: (i) both segments 1 and 2 fail to distribute entanglement; probability is $q^{2}$, (ii) both segments 1 and 2 succeed, but swapping them fails; probability is $(1-a) p^{2}$. The total probability is $q^{2}+(1-a) p^{2}$. On the other hand, we can stay in the state 100 in only one way - when segments 2 and 3 fail to distribute entanglement, which happens with probability $q^{2}$. Had we chosen to swap segments 2 and 3 first, the corresponding probabilities for these two transitions would have to be, well, swapped.

For the limit of the ratio of the standard deviation to the average value we now have

$$
\lim _{p \rightarrow 0} \frac{\sigma_{3}^{(\mathrm{dyn})}}{\bar{K}_{3}^{(\mathrm{dyn})}}=\frac{\sqrt{12 a^{4}-76 a^{3}-59 a^{2}+198 a+121}}{2 a^{2}+9 a+11} .
$$

For small $a$ this ratio is close to 1 , and for large $a$ it is $\approx 7 / 11 \approx 0.64$, as in the fixed-scheme case. The ratio of the average value of the dynamical scheme to that of the fixed scheme is shown in Fig. 4. We see that the dynamical scheme is slightly better, since we do not have

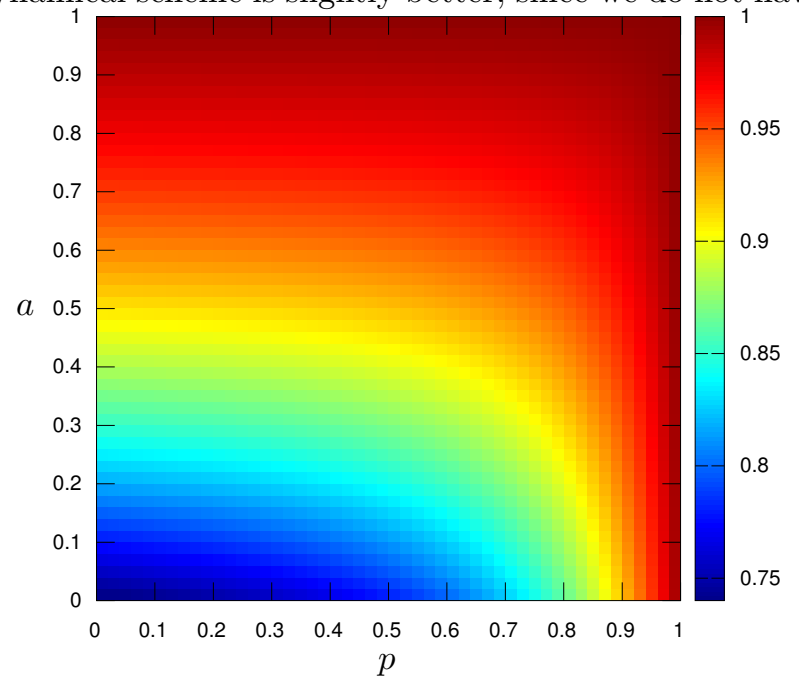

FIG. 4: The ratio of the dynamical to fixed waiting times for a 3 -segment repeater.

to wait for concrete segments and can start to swap them when they are ready. For $a=1$ or for $p=1$ the two expressions for the average value $(58)$ and $(61)$ coincide (note that for $a=1$ both expressions coincide with (2) for $n=3$ ), so the repeater rates are the same in this case. For small $p$ and $a$, we have

$$
\lim _{p, a \rightarrow 0} \frac{\bar{K}_{3}^{(\mathrm{dyn})}}{\bar{K}_{3}}=\frac{11}{15},
$$

so for small probabilities the dynamical scheme is approximately $25 \%$ faster.

\section{F. Four-segment repeater}

The TPM $P_{4}$ of a 4 -segment repeater with the recursive scheme can be easily obtained with the algorithm (36). This is then effectively a fixed, doubling scheme (see Fig. 3 for $n=4$, right). For the average waiting time of this scheme we have 


$$
\bar{K}_{4}=\frac{2 a^{2} p^{4}(p-1)(2 p-3)-a\left(20 p^{5}-72 p^{4}+93 p^{3}-53 p^{2}+10 p+4\right)+3(3-2 p)^{2}\left(2 p^{2}-3 p+2\right)}{a^{2} p(2-p)\left(a p^{2}-(a+2) p+3\right)\left(-a p^{3}+4 p^{2}-6 p+4\right)} .
$$

For the ratio of the variance to the average value we have

$$
\lim _{p \rightarrow 0} \frac{\sigma_{4}}{\bar{K}_{4}}=\frac{\sqrt{-96 a^{3}-272 a^{2}-1728 a+2916}}{54-4 a} .
$$

Not surprisingly, for small $a$ this ratio is also close to 1. For large $a$, it is close to $41 / 125=0.328$. For four segments there are more dynamical schemes than there are for three segments, but the waiting time of each of them can be computed with our method. Another fixed scheme, which is not doubling, is illustrated by Fig. 3 for $n=4$, left.

\section{GEOMETRIC APPROXIMATION}

The PDF of the waiting time of a single segment is the classical geometric distribution, but for two and more segments this is no longer exactly true. However, we now show that even in this case the PDF can be well approximated by a geometric distribution with appropriately chosen parameters. This fact is easy to establish for the deterministic swapping case. In fact, the probability that a single segment will finish in $k$ or less steps is equal to $1-q^{k}$ (because

$$
1-q^{k}=p \frac{1-q^{k}}{1-q}=\sum_{i=0}^{k-1} p q^{i}
$$

or, more simply, the probability that a segment is not ready in $k$ steps is $q^{k}$ ), so the probability that $n$ segments will finish in $k$ or less steps is $\left(1-q^{k}\right)^{n}$. The probability $p_{k}=\mathbf{P}\left(K_{n}=k\right)$ that the $n$ segments will finish in exactly $k$ steps is then equal to

$$
p_{k}=\left(1-q^{k}\right)^{n}-\left(1-q^{k-1}\right)^{n},
$$

for all $k \geqslant 1$. Expanding the sums and making a simple transformation of the terms, we obtain

$$
p_{k}=q^{k-1} \sum_{j=1}^{n}(-1)^{j+1}\left(\begin{array}{l}
n \\
j
\end{array}\right) q^{(k-1)(j-1)}\left(1-q^{j}\right) .
$$

In the limit $k \rightarrow+\infty$ the sum on the right tends to $n(1-q)=n p$, so we have

$$
p_{k} \sim n p q^{k-1}
$$

so for large $k$ the probability $p_{k}$ can be approximated by the geometric distribution with ratio $q$.

Non-deterministic swapping requires more sophisticated tools. A matrix $A$ is now called nonnegative (positive) if all its elements are nonnegative (positive). A nonnegative matrix $A$ is called stochastic if all its row sums are equal to one. If at least one of these inequalities is strict, then $A$ is called substochastic. A square matrix $A$ is called primitive if some of its power $A^{n}$ are positive. The Perron-Frobenius Theorem [37] states that for any nonnegative primitive matrix $A$ there is a real eigenvalue $\lambda_{1}$ with multiplicity one such that all other eigenvalues $\lambda_{j}$ (which can be complex) are strictly smaller by absolute value, $\left|\lambda_{j}\right|<\lambda_{1}$ for $j \geqslant 2$. If, in addition, $A$ is stochastic, then $\lambda_{1}=1$. If $A$ is substochastic, then $\lambda_{1}<1$. It is easy to show that in the case of $a<1$ (non-deterministic swapping) the matrix $Q$ of any scheme is primitive and substochastic and thus is subject to the Perron-Frobenius Theorem. Substochasticity is obvious, so we need only to prove that it is also primitive.

We show that all elements of $Q^{2}$ are strictly positive. In fact, let $\left\{s_{1}, \ldots, s_{N}\right\}$ be the states (a subset of the full set of $F_{2 n+1}$ states) that implement the given scheme without zero-time transitions. As usually, we assume that the state $s_{1}=0 \ldots 0$ is initial. Then we have $\left(Q^{2}\right)_{i j}=\mathbf{P}\left(s_{i} \rightarrow s_{1}\right) \mathbf{P}\left(s_{1} \rightarrow s_{j}\right)+\ldots$, where dots stand for other terms, which are nonnegative. Since $i$ is not the absorbing state we have $\mathbf{P}\left(s_{i} \rightarrow s_{1}\right)>0$, because there is always a chance that the last swapping fails, be it a fixed scheme or a dynamical one (it is at this point that we need the assumption $a<1$ ), and from the initial state we can go to any other state with nonzero probability, so we also have $\mathbf{P}\left(s_{1} \rightarrow s_{j}\right)>0$. It follows that $\left(Q^{2}\right)_{i j}>0$.

Applying the Perron-Frobenius Theorem, we can order the eigenvalues of $Q$ as $\lambda_{1}>\left|\lambda_{2}\right| \geqslant \ldots \geqslant\left|\lambda_{N-1}\right|$, and let $\mathbf{v}_{1}, \ldots, \mathbf{v}_{N-1}$ be the corresponding linearly independent eigenvectors. We can express the vector $\mathbf{u}$ from the decomposition $(16$ as a linear combination of these eigenvectors,

$$
\mathbf{u}=c_{1}^{\prime} \mathbf{v}_{1}+\ldots+c_{N-1}^{\prime} \mathbf{v}_{N-1} .
$$

We just have to solve the linear system $V \mathbf{c}^{\prime}=\mathbf{u}$, where $V$ is the matrix whose columns are the eigenvectors $\mathbf{v}_{j}$, which is nondegenerate due to the linear independence of $\mathbf{v}_{j}$. We then have

$$
\mathbf{p}_{k}=Q^{k-1} \mathbf{u}=c_{1}^{\prime} \lambda_{1}^{k-1} \mathbf{v}_{1}+\ldots+c_{N-1}^{\prime} \lambda_{N-1}^{k-1} \mathbf{v}_{N-1} .
$$

We need only the first element $p_{k}$ of this vector, so we finally obtain

$$
p_{k}=c_{1} \lambda_{1}^{k-1}+\ldots+c_{N-1} \lambda_{N-1}^{k-1}
$$

where $c_{j}=c_{j}^{\prime} v_{j}^{0}$ and $v_{j}^{0}$ is the first element of $\mathbf{v}_{j}$. For large $k$, we clearly have

$$
p_{k} \approx c_{1} \lambda_{1}^{k-1}
$$

because the largest eigenvalue $\lambda_{1}$ and its corresponding eigenvector are unique. Compare this expression with 


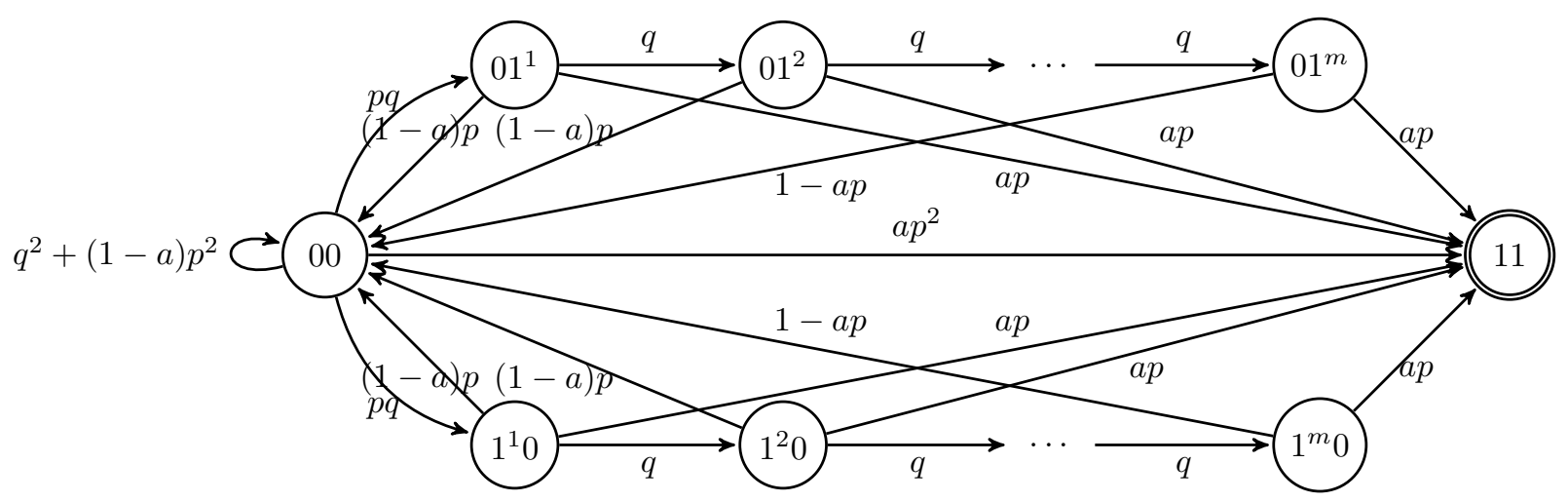

FIG. 5: Markov chain of a two-segment repeater with finite memory cutoff and arbitrary swapping probability.

Eq. (69), where it is possible to find $\lambda_{1}=q$ and $c_{1}=n p$ explicitly. In both cases, starting from some sufficiently large $k$ the probabilities $p_{k}$ are well described by a geometric distribution, similar to those for a single repeater segment. To our knowledge, this has never been shown explicitly. In fact, the common wisdom seems to be that not only a single segment, but an entire quantum repeater follows exactly a geometric distribution. We showed that this holds only approximately.

\section{FINITE MEMORY}

Let us first show that the Markov chain method can reproduce Eq. (1), which gives the waiting time of a twosegment repeater with non-deterministic swapping and arbitrary memory cutoff. We introduce auxiliary states $01^{i}$ and $1^{i} 0, i=1, \ldots, m$. The superscript denotes the time the segment waits in the ready state. When this time exceeds the limit, $m$ time units, the segment is forcefully reset to the initial state. The corresponding Markov chain is shown in Fig. 5. It is easy to construct the TPM corresponding to this chain and, with the help of a computer algebra system, to verify that this TPM leads to Eq. (1) for the average waiting time.

In the following, we show how to apply the Markov chain approach to repeaters with deterministic swapping, $a=1$, and finite memory cutoff, $m$. In this case, we need another full set of states describing such a quantum repeater and Markov chains with several absorbing states. The states are the tuples $\left(i_{1}, \ldots, i_{n}\right)$ with $i_{j}=0, \ldots, m$ such that there is at least one $j=1, \ldots, n$ with $i_{j}=0$ or $i_{j}=1$. Totally, there are $N=(m+1)^{n}-(m-1)^{n}$ such states. Each component $i_{j}$ denotes the time passed since the $j$-th segment has successfully distributed entanglement. The condition that there must be a component with value 0 or 1 means that a valid state is one that is either not ready (some component is 0) or has just become ready (no zero components but at least one is equal to 1). Tuples with all components larger than 1 are not needed - they describe those states where all the segments are in the ready state for longer than necessary. Tuples with at least one zero component are non-absorbing states, and all the others are absorbing. There are $N_{0}=(m+1)^{n}-m^{n}$ non-absorbing states and $N_{1}=m^{n}-(m-1)^{n}$ absorbing ones. If we follow our usual convention and put all absorbing states at the end, then the PTM of this Markov chain can be decomposed as follows:

$$
P=\left(\begin{array}{cc}
Q & U \\
0 & I
\end{array}\right),
$$

where $Q$ is an $N_{0} \times N_{0}$ matrix (whose elements are probability transitions between non-absorbing states), $U$ is an $N_{0} \times N_{1}$ matrix, 0 is the zero $N_{1} \times N_{0}$ matrix and $I=I_{N_{1}}$ is the $N_{1} \times N_{1}$ identity matrix. As before, $R=(I-Q)^{-1}$ is the fundamental matrix of the chain (where $I=I_{N_{0}}$ is the $N_{0} \times N_{0}$ identity matrix). According to the general result, Ref. [35, the absorption time as usual reads as $R \mathbf{1}$ (so it does not matter how many absorbing states there are). We only need to compute the TPM $P$.

If fact, we only need to compute the matrix $Q$. Consider a non-absorbing state $\left(i_{1}, \ldots, i_{n}\right)$. Denote by $n_{z}=$ $\left|\left\{j \mid i_{j}=0\right\}\right|$ the number of its zero components. Since the state is non-absorbing, $n_{z}>0$. If for some $j$ we have $i_{j}=m$, i.e., at least one segment has reached the maximal allowed time, then such a state can go only into two other states: to the absorbing state $\left(i_{1}^{\prime}, \ldots, i_{n}^{\prime}\right)$ (where $i_{j}^{\prime}=i_{j}$ if $i_{j}>0$ and $i_{j}^{\prime}=1$ if $\left.i_{j}=0\right)$ with probability $p^{n_{z}}$, or to the initial state $(0, \ldots, 0)$ with probability $1-p^{n_{z}}$. If none of the segments have reached the maximal time, then

$$
\begin{aligned}
& \mathbf{P}\left(\left(i_{1}, \ldots, i_{n}\right) \rightarrow\left(i_{1}^{\prime}, \ldots, i_{n}^{\prime}\right)\right)= \\
& \mathbf{P}\left(i_{1} \rightarrow i_{1}^{\prime}\right) \ldots \mathbf{P}\left(i_{n} \rightarrow i_{n}^{\prime}\right),
\end{aligned}
$$

where $\mathbf{P}(0 \rightarrow 0)=1-p, \mathbf{P}(0 \rightarrow 1)=p, \mathbf{P}(i \rightarrow i+1)=1$, and all other probabilities are zero. It is straightforward to construct this TPM programmatically in a computer algebra system for given parameters $p, n$ and $m$, where, recall, we have set $a=1$. With the help of such a system, it is easy to verify that the average waiting time produced by this TPM gives exactly the expression (3), $\bar{K}_{n}^{(m)}$, i.e., the average waiting time for deterministic swapping and 
finite memory cutoff. Our formalism can also be used to treat the most general case of arbitrary $a$ and $m$.

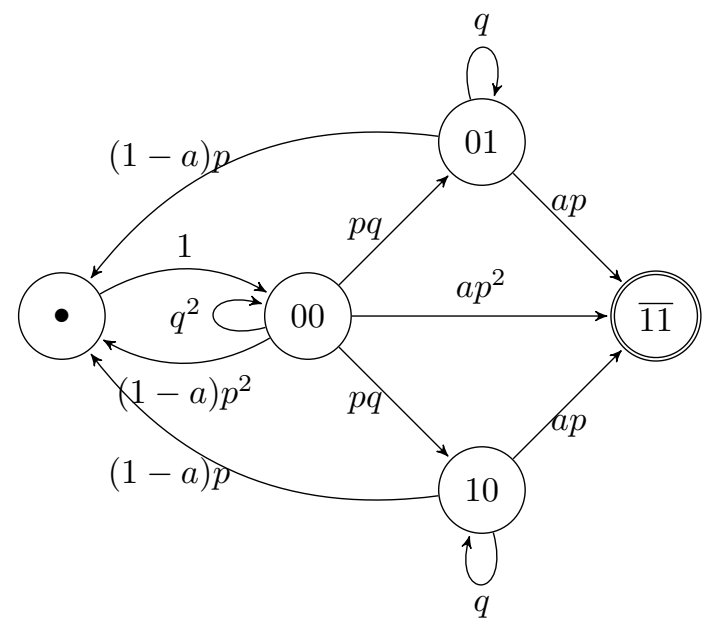

FIG. 6: Markov chain of a two-segment repeater with classical communication.

\section{CLASSICAL COMMUNICATION}

In this section we add classical communication to the basic scheme considered before. Note that classical communication times in a general quantum repeater with probabilistic swapping have been considered before, e.g. in Ref. 25, however, once again, those existing schemes are approximate depending on the usual assumptions, as discussed in Sec. II. We assume that the number of segments is a power of two, $n=2^{d}$, and on each level $i=2, \ldots d$ it typically takes $2^{i-1}$ units of time to reinitiate the segments in the case of swapping failure (because $\left.2^{i} / 2=2^{i-1}\right)$. We also need classical communication to communicate success of a swapping operation, but for simplicity we only consider the case of failure. The idea is to introduce additional states that correspond to these additional $2^{i-1}$ time units. In the case of $d=1$ we need one additional state, which we denote by dot. The corresponding Markov chain of a two-segment repeater with classical communication is shown in Fig. 6. Each transition in this figure takes exactly one unit of time. The corresponding TPM reads as

$$
P=\left(\begin{array}{ccccc}
q^{2} & p q & p q & (1-a) p^{2} & a p^{2} \\
0 & q & 0 & (1-a) p & a p \\
0 & 0 & q & (1-a) p & a p \\
1 & 0 & 0 & 0 & 0 \\
0 & 0 & 0 & 0 & 1
\end{array}\right)
$$

We now show how to construct TPMs for larger repeaters.

We describe an algorithm to "double" a given repeater with the TPM $P$ and the classical communication time of $c$ time units. The original repeater can employ any kind of scheme. Applying our algorithm recursively all the way down, we can compute the TPM of a repeater with $n=2^{d}$ segments and classical communication at all levels.

Given a quantum repeater with the TPM $P$, we want to construct a quantum repeater of double length, performing the last swapping exactly in the middle of the new repeater, and assuming that restarting the process in the case of failure takes $c$ time units. If the original repeater is described by the states $s_{1}, \ldots, s_{N}$, the doubled repeater without classical communication is described by the $N^{2}$ states $s_{i} s_{j}, i, j=1, \ldots, N$. To include the classical communication into the scheme we need $c$ additional states, which we denote as $\bullet_{1}, \ldots, \bullet_{c}$, so the full set of states contains $N^{2}+c$ elements. We arrange these states in the following order: $s_{i} s_{j}$ for all $i, j$ except the state $s_{N} s_{N}$, then $\bullet_{i}, i=1, \ldots, c$, and finally $s_{N} s_{N}$. The "doubled" TPM $P_{d}$ can then be partitioned into nine blocks as

$$
P_{d}=\left(\begin{array}{ccc}
P_{s s} & P_{s \bullet} & P_{s N} \\
P_{\bullet s} & P_{\bullet \bullet} & P_{\bullet N} \\
0 & 0 & 1
\end{array}\right) .
$$

We give explicit expressions the first six blocks. Let partition $P \otimes P$ be as follows:

$$
P \otimes P=\left(\begin{array}{cc}
Q_{d} & \mathbf{u}_{d} \\
0 & 1
\end{array}\right) .
$$

Then we simply have $P_{s s}=Q_{d}$ since the transition probability between non-absorbing states $s_{i_{1}} s_{j_{1}}$ and $s_{i_{2}} s_{j_{2}}$ is the product of transition probabilities $s_{i_{1}} \rightarrow s_{i_{2}}$ and $s_{j_{1}} \rightarrow s_{j_{2}}$. For the next block we have

$$
P_{s \bullet}=\left(\begin{array}{l|l}
(1-a) \mathbf{u}_{d} & \ldots \\
\ldots
\end{array}\right),
$$

because when the last swapping fails, we have to go to the state $\bullet_{1}$ and then restart the process through the sequence of transitions $\bullet_{1} \rightarrow \ldots \rightarrow \bullet_{c} \rightarrow s_{1} s_{1}$. Each of these transitions happens with probability one, so we also have

$$
P_{\bullet s}=\left(\begin{array}{ccccc}
0 & 0 & \ldots & 0 & 0 \\
0 & 0 & \ldots & 0 & 0 \\
\ldots & \ldots & \ldots & \ldots & \ldots \\
0 & 0 & \ldots & 0 & 0 \\
1 & 0 & \ldots & 0 & 0
\end{array}\right), P_{\bullet \bullet}=\left(\begin{array}{ccccc}
0 & 1 & \ldots & 0 & 0 \\
0 & 0 & \ldots & 0 & 0 \\
\ldots & \ldots & \ldots & \ldots & \ldots \\
0 & 0 & \ldots & 0 & 1 \\
0 & 0 & \ldots & 0 & 0
\end{array}\right) .
$$

The other blocks are just $P_{s N}=a \mathbf{u}_{d}$ and $P_{\bullet}=\mathbf{0}$. This gives us the full TPM of the doubled repeater with classical communication. One can easily check that the matrix 76. can be obtained from the matrix (37) with this algorithm with $c=1$. Fig. 7 illustrates the effect of classical communication as the ratio of the averaged waiting time with classical communication to that without. One can see that the maximal effect of classical communication grows with the number of segments, and also, as one 

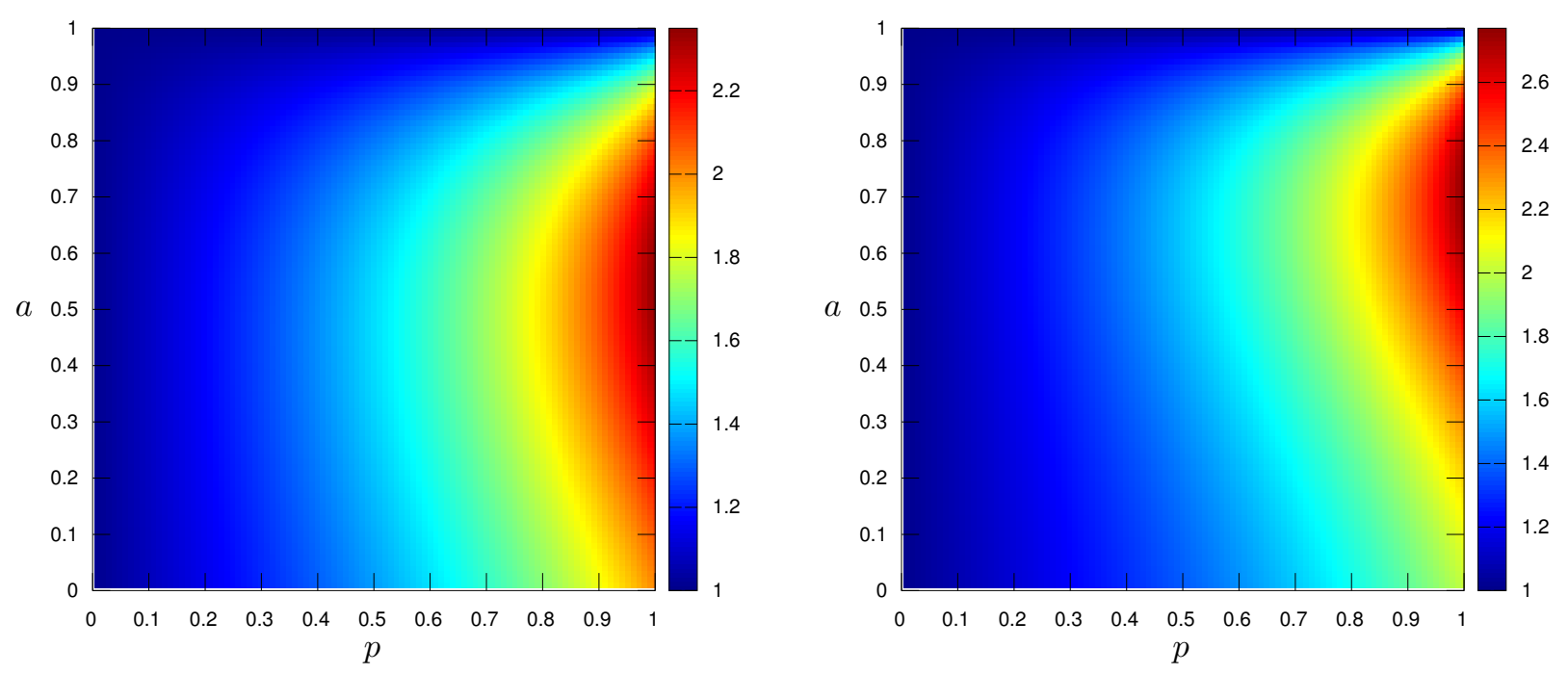

FIG. 7: The ratio of the waiting time with classical communication to the waiting time without for $n=8$ (left) and $n=16$ (right) segments.

would expect, for larger probabilities $p$ and $a$ where the absolute waiting times decrease and so the net effects of classical communication become more pronounced. For small $p$ and $a$, waiting times are so long anyway that the addition of classical communication times hardly makes a difference. This is why in the regime of the commonly used approximation, the classical communication times are typically neglected on higher levels. However, for a practical quantum repeater with moderate $p$ and significantly larger $a$, for many segments, the classical communication should be included. In the case $a=1$, it is already implicitly included via the elementary time unit for the initial entanglement distribution. Note that once memory cutoffs and even explicit memory errors (such as dephasing) are included, classical communication times become relevant even for small repeaters.

\section{GENERATING FUNCTIONS}

An alternative approach to obtain the average waiting time is to use the method of generating functions 38 . In general, this method is inferior to the Markov chain method, since numerically it is much easier to deal with matrices and vectors than with functions. On the other hand, generating functions can be useful in more complicated situations, where the Markov chain approach cannot be easily applied. We shall give an example for this in this section.

If $X$ is a random variable taking only non-negative integer values, then its probability generating function (PGF) $g(t)$ is defined by

$$
g(t)=\sum_{k=0}^{+\infty} \mathbf{P}(X=k) t^{k} .
$$

Any PGF $g(t)$ satisfies the equality $g(1)=1$. The PGF $g(t)$ contains full statistical information about the corresponding random variable $X$, for example, the average value $\bar{X}$ and the variance $\sigma^{2}=\overline{X^{2}}-\bar{X}^{2}$ via $\bar{X}=g^{\prime}(1)$ and $\sigma^{2}=g^{\prime \prime}(1)+g^{\prime}(1)-g^{\prime}(1)^{2}$. We now show how to reproduce Theorem 1 .

Consider a chain with $N$ states $s_{i}, i=1, \ldots, N$, and single absorbing state $s_{N}$. For any state $i=1, \ldots, N$ we introduce an integer-valued random variable $K_{i}$, which is defined to be the number of steps it takes to get to the absorbing state starting in the state $s_{i}$ (note that $K_{N}=0$ since we are already in the absorbing state). Let $g_{i}(t)$ denote the PGF of $K_{i}$ and $\mathbf{g}(t)=\left(g_{1}(t), \ldots, g_{N-1}(t)\right)^{\mathrm{T}}$ be the vector of these PGFs. They are connected by the following system of linear equations

$$
g_{i}(t)=\sum_{j=1}^{N} p_{i j} t_{i j} g_{j}(t),
$$

where $t_{i j}=t$ if the transition $s_{i} \rightarrow s_{j}$ takes one time unit and $t_{i j}=1$ if $s_{i} \rightarrow s_{j}$ is a zero-time transition. It is clear that $g_{N}(t)=1$. In the matrix notation it reads as

$$
\mathbf{g}(t)=Q(t) \mathbf{g}(t)+\mathbf{u}(t),
$$

where $Q(t)$ is the matrix $Q$ when each element is multiplied by $t$ except zero-time transitions elements, which are left unchanged. The vector $\mathbf{u}(t)$ is obtained in a similar way from $\mathbf{u}$, where $Q$ and $\mathbf{u}$ are defined by the decomposition (16). The solution of this system reads as

$$
\mathbf{g}(t)=(E-Q(t))^{-1} \mathbf{u}(t) .
$$

This expression can be applied to an arbitrary absorbing chain with a single absorbing state and zero-time transitions. In the case of a Markov chain without zero-time transitions all elements are multiplied by $t$, so we have $Q(t)=t Q, \mathbf{u}(t)=t \mathbf{u}$, and the solution becomes

$$
\mathbf{g}(t)=t(E-t Q)^{-1} \mathbf{u} .
$$


It is easy to see that

$$
\mathbf{g}(1)=(E-Q)^{-1} \mathbf{u}=(E-Q)^{-1}(E-Q) \mathbf{1}=\mathbf{1}
$$

so this solution satisfies the basic property of a PGF. The derivative $\mathbf{g}^{\prime}(t)$ is easy to compute. We have

$$
(E-t Q) \mathbf{g}(t)=t(E-Q) \mathbf{1}
$$

Differentiating this relation we get

$$
-Q \mathbf{g}(t)+(E-t Q) \mathbf{g}^{\prime}(t)=(E-Q) \mathbf{1}
$$

Substituting $t=1$ and taking into account that $\mathbf{g}(1)=\mathbf{1}$ we obtain the relation

$$
\overline{\mathbf{K}}=\mathbf{g}^{\prime}(1)=(E-Q)^{-1} \mathbf{1}=R \mathbf{1} .
$$

The second moment can be computed as

$$
\overline{\mathbf{K}^{\circ 2}}=\mathbf{g}^{\prime \prime}(1)+\mathbf{g}^{\prime}(1)=(2 R-I) \overline{\mathbf{K}},
$$

which coincides with Eq. (19).

We now compute the probabilities $\mathbf{p}_{k}$ using the generating functions approach. Let us denote $\tilde{\mathbf{p}}_{k}=\mathbf{P}(\mathbf{K}>$ $k$ ) and introduce the corresponding generating function (GF)

$$
\tilde{g}(t)=\sum_{k=0}^{+\infty} \tilde{\mathbf{p}}_{k} t^{k}
$$

It is easy to see that the standard PGF can be expressed in terms of the standard generating function as

$$
g(t)=1-(1-t) \tilde{g}(t)
$$

For the random variables under discussion we explicitly get this GF from Eq. 85

$$
\tilde{\mathbf{g}}(t)=(E-t Q)^{-1} \mathbf{1} .
$$

For the $k$-th derivative we have

$$
\tilde{\mathbf{g}}^{(k)}(t)=k !(E-t Q)^{-(k+1)} Q^{k} \mathbf{1},
$$

from which we immediately obtain the probabilities

$$
\tilde{\mathbf{p}}_{k}=\frac{\tilde{\mathbf{g}}^{(k)}(0)}{k !}=Q^{k} \mathbf{1}
$$

The probabilities $\mathbf{p}_{k}$ can be computed as

$$
\mathbf{p}_{k}=\tilde{\mathbf{p}}_{k-1}-\tilde{\mathbf{p}}_{k}=Q^{k-1}(E-Q) \mathbf{1}=Q^{k-1} \mathbf{u},
$$

which is exactly the expression 18 .

As a concrete example, let us compute the PGF of a two-segment repeater. According to Eq. (15), the matrix $Q$ and the vector $\mathbf{u}$ read as

$$
\begin{aligned}
Q & =\left(\begin{array}{ccc}
q^{2}+(1-a) p^{2} & p q & p q \\
(1-a) p & q & 0 \\
(1-a) p & 0 & q
\end{array}\right), \\
\mathbf{u} & =\left(a p^{2}, a p, a p\right)^{\mathrm{T}} .
\end{aligned}
$$

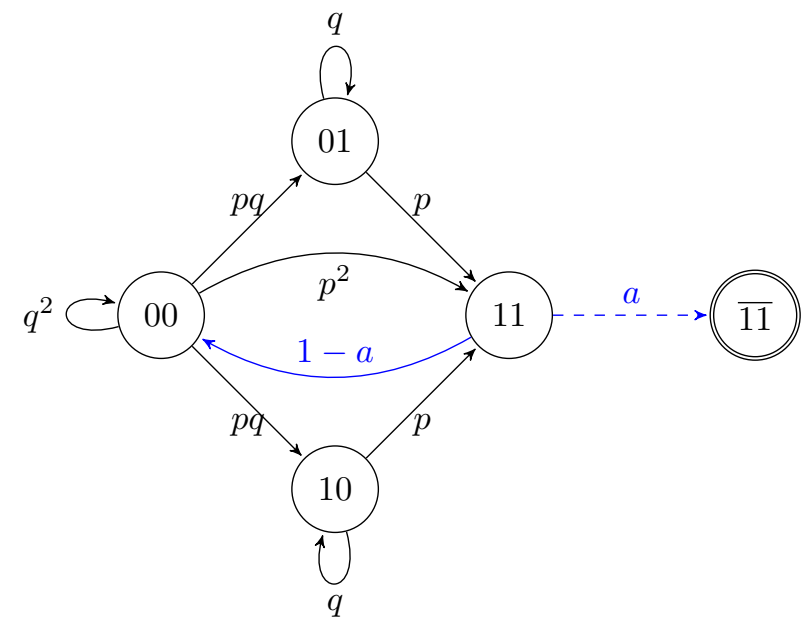

FIG. 8: The Markov chain for a two-segment repeater with different time scales.

From Eq. 85 we obtain

$$
g(t)=\frac{a p^{2} t(1+q t)}{1-\left(2-3 p+(2-a) p^{2}\right) t+q\left(1-2 p+a p^{2}\right) t^{2}},
$$

where $g(t)$ is the first element of the vector $\mathbf{g}(t)$. It is easy to check that $g^{\prime}(1)=\bar{K}_{2}$ and

$$
g^{\prime \prime}(1)+g^{\prime}(1)-g^{\prime}(1)^{2}=\sigma_{2}^{2},
$$

where the variance $\sigma_{2}^{2}$ is given by Eq. (56).

Up to now we have always assumed that all time intervals can be expressed in terms of one common time unit. Let us consider a more general problem, where restarting the process takes the time $\tau^{\prime}$, which is independent of $\tau$ (while, for simplicity, here we again ignore the classical communication times when the swapping succeeds, though this can be also easily included). Fig. 8 shows the Markov chain of such a repeater. All transitions marked by black arrows take the time $\tau$. The transition marked by a blue arrow takes the time $\tau^{\prime}$. The time for the transition marked by the blue dashed arrow (swapping success) is ignored. The absorption time now reads as

$$
T=K \tau+L \tau^{\prime}
$$

where the random variable $K$ is the number of black transitions and $L$ is the number of blue transitions.

We mark different transitions by different variables $(t$, as before, marks attempts to distribute entanglement and $v$ marks unsuccessful attempts to perform swapping corresponding to the blue line in the figure). The matrix $Q$ with this information included reads as

$$
Q(t, v)=\left(\begin{array}{cccc}
t(1-p)^{2} & t p(1-p) & t p(1-p) & t p^{2} \\
0 & t(1-p) & 0 & t p \\
0 & 0 & t(1-p) & t p \\
v(1-a) & 0 & 0 & 0
\end{array}\right)
$$


The PGFs of the absorption times are given by

$$
\begin{aligned}
& \mathbf{g}(t, v)=(E-Q(t, v))^{-1} \mathbf{u}(t, v), \\
& \mathbf{u}(t, v)=(0,0,0, a)^{\mathrm{T}} .
\end{aligned}
$$

For the first component, $g=g(t, v)$, we have

$$
g(t, v)=\frac{a p^{2} t(1+q t)}{1-\left(2-3 p+p^{2}(1+(1-a) v)\right) t+q\left(1-2 p+p^{2}(1-(1-a) v)\right) t^{2}} .
$$

If we set $v=1$ in this expression, i.e. if we ignore the additional information, we get the less detailed PGF given by Eq. (98). Taking into account that

$$
\bar{K}=g_{t}^{\prime}(1,1), \quad \bar{L}=g_{v}^{\prime}(1,1),
$$

we obtain

$$
\bar{T}=\bar{K}_{2} \tau+\frac{1-a}{a} \tau^{\prime} .
$$

The variance $\sigma^{2}$ can be computed in the usual way, and the only new part is computing the correlation

$$
\overline{K L}=g_{t v}^{\prime \prime}(1,1)=\frac{2(1-a)(3-2 p)}{a^{2}(2-p) p} .
$$

It is easy to see that for small $p$ and $a$ the main contribution to $\bar{T}$ is given by the first term, $\bar{K}_{2} \tau$.

\section{LUMPABILITY}

The full set of states needed to describe an $n$-segment quantum repeater with infinite memory is $F_{2 n+1}=$ $\mathcal{O}\left(2.61 \ldots{ }^{n}\right)$. The doubling scheme requires less states, namely $2^{n}$. Here we show that if the scheme has some symmetry, this symmetry can be exploited to greatly reduce the number of states 39 . We emphasize that the method presented here is just an implementation trick and in no way influences the result produced by the original algorithm. It just makes this algorithm much more practical than it otherwise would be.

The basic idea of the trick is the observation that in the absorption time problem we are interested only in the number of steps from an initial state to the (or a) absorbing state. If, on the way to absorption, some intermediate states give the same contribution to the number of states, it makes sense to combine them into one large state and work with this more coarse Markov chain. This idea to replace the original Markov chain by a coarser one which still correctly describes the desired property (the number of steps in this case) is formalized by the lumpability property.

Let the states of the Markov chain be $s_{1}, \ldots, s_{N}$. Consider a partition of this set of states,

$$
\left\{s_{1}, \ldots, s_{N}\right\}=S_{1} \cup \ldots \cup S_{M},
$$

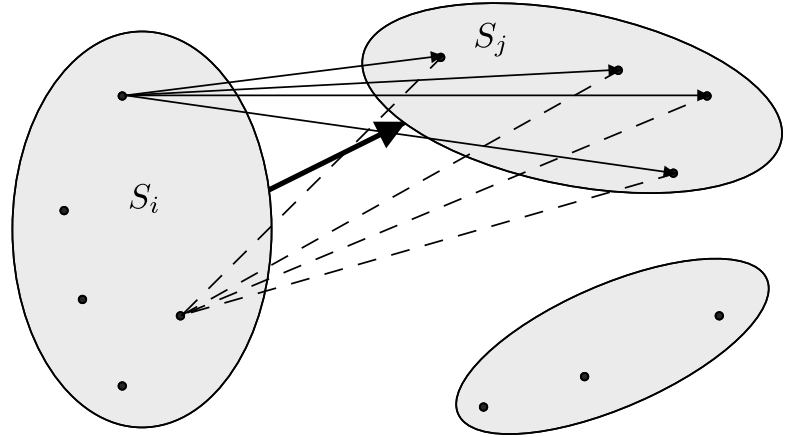

FIG. 9: Illustration of the lumpability condition.

so that $S_{i} \cap S_{j}=\varnothing$ for $i \neq j$. If this partition satisfies the condition that the probability

$$
\mathbf{P}\left(S_{i} \rightarrow S_{j}\right)=\sum_{s_{l} \in S_{j}} \mathbf{P}\left(s_{m} \rightarrow s_{l}\right)
$$

does not depend on $s_{m} \in S_{i}$, then the chain is called lumpable with respect to the partition (107). This is illustrated by Fig. 9, where the sums of solid and dashed lines must be equal and their common value is considered to be the new probability transition, denoted by the bold line. We can construct a new Markov chain with the states $S_{1}, \ldots, S_{M}$ and the transition probabilities between them are given by Eq. (108). The new, coarser Markov chain has fewer states and thus is easier to deal with, so if it preserves the information we need, we can work directly with this new chain instead of the original, larger one. We now give two concrete examples of lumpability of Markov chains in the context of quantum repeaters.

\section{A. Deterministic swapping}

Consider a general quantum repeater with $n$ segments (which is not necessarily a power of two) with deterministic swapping. In a previous section we studied the properties of such a repeater with a Markov chain with $2^{n}$ states, which correspond to $n$-digit binary numbers. We show that it is possible to compress this chain to the size of $n+1$ and it will still contain the complete information about the waiting time of the corresponding repeater. Because swapping never fails, all states with 
the same number of ready segments are equivalent. Let $S_{i}, i=0,1, \ldots, n$, be the set of all $n$-binary digits with exactly $i$ components equal to one. Obviously, these sets form a partition of the quantum repeater's set of states. The transition probabilities read as

$$
\mathbf{P}\left(S_{i} \rightarrow S_{j}\right)=\left\{\begin{array}{ll}
0 & j<i \\
\left(\begin{array}{c}
n-i \\
j-i
\end{array}\right) p^{j-i} q^{n-j} & i \leqslant j
\end{array} .\right.
$$

We claim that the average waiting time of the repeater that started in the state $S_{i}$ is given by

$$
\bar{K}(i)=\sum_{l=1}^{n-i}(-1)^{l+1}\left(\begin{array}{c}
n-i \\
l
\end{array}\right) \frac{1}{1-q^{l}},
$$

which coincides with Eq. (43) for any subset $I \subseteq[n]$ with $|I|=i$. We need to check that these functions satisfy the linear equations

$$
1+\sum_{j=i}^{n} \mathbf{P}\left(S_{i} \rightarrow S_{j}\right) \bar{K}(j)=\bar{K}(i) .
$$

This can be easily done with the help of the following symbolic relation:

$$
\sum_{j=0}^{n-i} \sum_{l=1}^{n-i-j}=\sum_{l=1}^{n-i} \sum_{j=0}^{n-i-l} .
$$

In this case the original Markov chain had a lot of symmetry, which was exploited to reduce its size from exponential with respect to the number of segments to a linear size. In the next subsection we show that a reduction in size is also possible with less symmetric chains, though to a lesser degree.

\section{B. Doubling}

Consider a quantum repeater with non-deterministic swapping and a power-of-two number of segments $n=2^{d}$ with the standard doubling scheme. Before we demonstrated that this scheme requires a number of $N_{n}=2^{n}$ states. This size is better than the number of all states, $\mathcal{O}\left(2.61 \ldots{ }^{n}\right)$, but it is still impractical for $n>8$. We now show that we can describe this scheme by a Markov chain with $\mathcal{O}\left(1.34 \ldots{ }^{n}\right)$ states. This is still exponential with respect to the number of segments, but it allows one to compute the exact rate of a quantum repeater with up to 32 segments.

Let us start with the simplest, 2-segment repeater. The doubling scheme in this case requires four states: $s_{1}=00$, $s_{2}=01, s_{3}=10$ and $s_{4}=\overline{11}$. Because the two halves of the repeater are independent in this scheme, the states 01 and 10 are equivalent and can be grouped into one class. So, instead of four states, three are enough: $S_{1}=\{00\}$, $S_{2}=\{01,10\}$ and $S_{3}=\{\overline{11}\}$. The corresponding Markov chain is shown in Fig. 10. The 4-segment repeater can

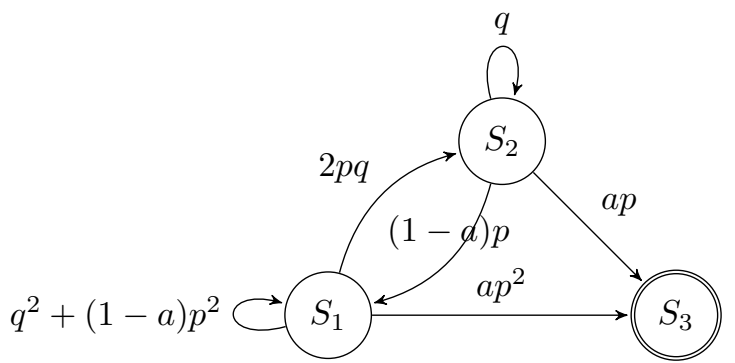

FIG. 10: The compressed Markov chain of a 2-segment repeater.

thus be described by nine states $S_{i} S_{j}, i, j=1, \ldots, 3$ instead of sixteen $s_{i} s_{j}, i, j=1, \ldots, 4$. But we can go even further: the states $S_{i} S_{j}$ for $i \neq j$ are equivalent and can be combined into one group, so six states are enough. These states are $\left\{S_{1} S_{1}\right\},\left\{S_{1} S_{2}, S_{2} S_{1}\right\},\left\{S_{1} S_{3}, S_{3} S_{1}\right\}$, $\left\{S_{2} S_{2}\right\},\left\{S_{2} S_{3}, S_{3} S_{2}\right\}$, and $\left\{S_{3} S_{3}\right\}$. This process is repeated on higher levels. Instead of the recursive relation $N_{2 n}=N_{n}^{2}$, we now have the relation

$$
N_{2 n}=\frac{1}{2} N_{n}\left(N_{n}+1\right) .
$$

There is no exact expression for the numbers defined by this recursive rule, but they can be well approximated as $N_{n} \approx 2 \cdot 1.34 \ldots{ }^{n}$ [40]. For $n=32$ the original Markov chain would have more than $4 \cdot 10^{9}$ states, while the new, compressed chain has only 26796 states. Solving the corresponding system of linear equations requires around 20 $\mathrm{Gb}$ of RAM and takes approximately one minute of time on a modern 8-core processor. Direct treatment of the original chain is intractable with the current technology.

\section{VERIFICATION}

Here we present a simple algorithm for Monte-Carlo simulation of a quantum repeater with a power-of-two number of segments, $n=2^{d}$, and a doubling scheme, which will allow us to validate our analytical results obtained before. First, we need a function that simulates a single entanglement distribution over the quantum repeater. Its input parameters are the level $d$, probabilities $p$ and $a$ and the boolean parameter $c c$ which says whether to include classical communication or not. The output is the number of steps it took to distribute entanglement in this concrete run, so the output will vary from run to run.

$$
\begin{aligned}
& \text { function } \mathrm{K}(d, p, a, c c) \\
& \begin{array}{l}
k \leftarrow 0 \\
\text { if } d=0 \text { then } \\
\text { while true do } \\
k \leftarrow k+1 \\
\quad \text { if RAND }<p \text { then }
\end{array}
\end{aligned}
$$




\author{
break \\ end if \\ end while \\ else \\ while true do \\ $k_{1} \leftarrow \mathrm{K}(d-1, p, a, c c)$ \\ $k_{2} \leftarrow \mathrm{K}(d-1, p, a, c c)$ \\ $k \leftarrow k+\max \left(k_{1}, k_{2}\right)$ \\ if RAND $<a$ then \\ break \\ else \\ if $c c$ then \\ end if \\ end if \\ end while \\ end if \\ return $k$

\section{end function}

The function RAND here is a generator of pseudorandom numbers, uniformly distributed in the interval $[0,1]$. The function $\mathrm{K}$ implements the random variable $K$. To obtain the average waiting time, we need to call this function many times and compute the average value of its output. One can also compute the variance and other higher moments. Using this simple function, it is possible to verify that our analytical results are in very good agreement with the results of the simulation.

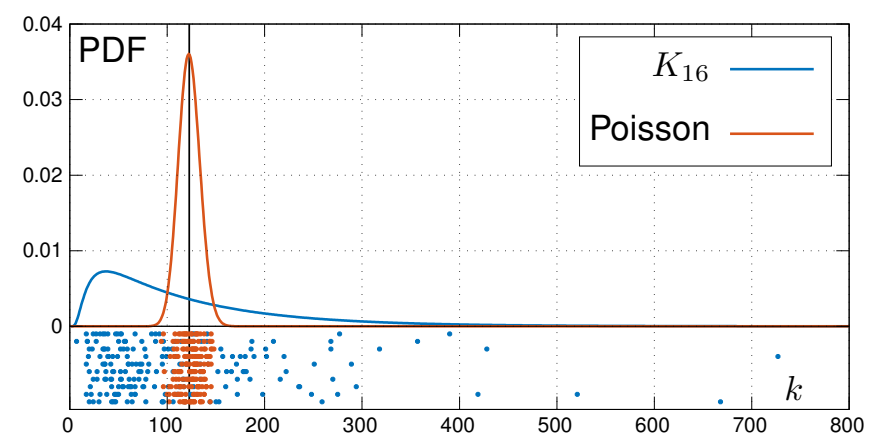

FIG. 11: The PDF of $K_{16}$ (blue) with $p=a=0.5$ vs. the Poisson PDF (red) with the same average value $\bar{K}_{16}$ (vertical).

This function can also be used to demonstrate the large variance of the waiting time. The results of calling it 200 times (10 series of experiments with 20 runs each) for a 16-segment quantum repeater are shown in the lower part of Fig. 11. For comparison, we also plotted the same number of steps generated with a Poisson random number generator with the same average. We can see the dramatic difference in the behavior of the two random variables. The Poisson variable is compactly located around its average. On the other hand, the wait- ing time varies widely around the average, and even in such a small number of experiments one can expect much shorter or much longer waiting times. The short times are not problematic, but long waiting times can lead to failures of memory-based components. The upper half of the figure shows the distributions of these random variables. The Poisson distribution has a well-known bell shape, while the waiting time distribution significantly deviates from this shape. As a consequence, its average value alone is not sufficient to give an acceptable characteristic of the waiting time random variable.

\section{LARGE REPEATERS}

The Markov chain approach developed above can give an exact result for small repeaters $(n \sim 2-4)$, a numerical result for medium-size repeaters $(n \sim 4-32)$, but it is intractable for large repeaters $(n>32)$. For the latter class of repeaters, we develop a family of approximations, where we can trade simplicity for accuracy and vice versa.

Generally, approximations are not necessary if we can compute exact values, but they are unavoidable when we cannot obtain the result precisely. A simple way to get an approximation in the power-of-two case $n=2^{d}$ is to consider an $n$-segment repeater as a $n / 2$-segment one where each segment of the new repeater is a pair of the old repeater's segments. Each new segment can be assigned an effective probability $p^{\prime}=1 / \bar{K}_{2}$, and we have an approximate relation

$$
\bar{K}_{n}(p, a) \approx \bar{K}_{\frac{n}{2}}\left(\frac{1}{\bar{K}_{2}(p, a)}, a\right) .
$$

This scheme can be repeated and finally we have an approximation

$$
\bar{K}_{2^{d}}(p, a) \approx \bar{K}_{2}\left(\frac{1}{\bar{K}_{2}\left(\frac{1}{\bar{K}_{2}(\ldots)}, a\right)}, a\right),
$$

where on the right-hand side there are $d$ nested $\bar{K}_{2}$ 's. A similar approach is used in Ref. 41] and also $\bar{K}_{n}^{\prime}$ in Eq. (9) is based on this kind of approach, however, with the lowest-level waiting time approximated for small $p$. If $\bar{K}_{2}$ is the only average waiting time that we know, then this approximation is the best that we can construct. If we know other averages $\bar{K}_{4}, \bar{K}_{8}, \ldots$, then we can construct other, better approximations. For example, we have

$$
\bar{K}_{16}(p, a) \approx \bar{K}_{8}\left(\frac{1}{\bar{K}_{2}(p, a)}, a\right) \approx \bar{K}_{4}\left(\frac{1}{\bar{K}_{4}(p, a)}, a\right),
$$

and several others, where $\bar{K}$ 's subindices are $2|8,2| 4 \mid 2$, $2|2| 4,2|2| 2 \mid 2$ and $4|2| 2$. To compare the quality of different approximations, we shall use the quantity

$$
E=100\left|\frac{\text { approx. }}{\text { exact }}-1\right| \text {, }
$$



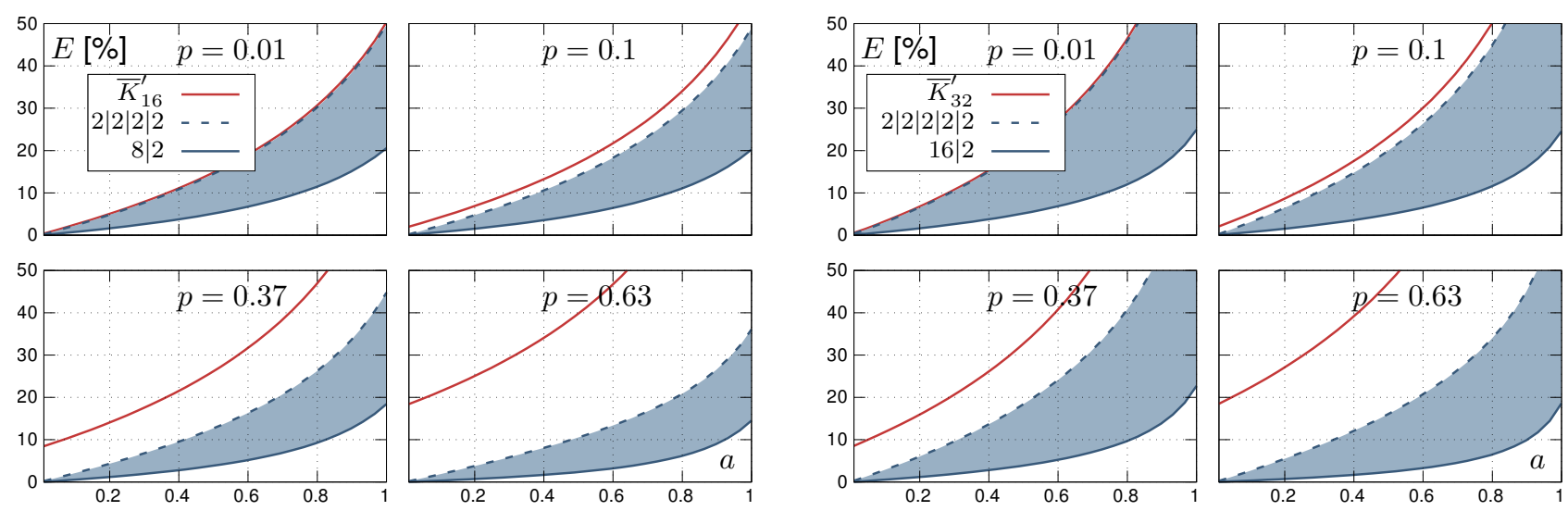

FIG. 12: The relative error of different approximations as a function of $a$ for some values of $p$, with $n=16$ (left) and $n=32$ (right) segments.

which is the relative error measured in percents. In Fig. 12 we plot these approximations for some practical values of $p=e^{-L_{0} / L_{\text {att }}}$ with $L_{\text {att }}=22 \mathrm{~km}$ (corresponding to $L_{0}=100,50,22$, and $10 \mathrm{~km}$, respectively, assuming deterministic local state preparations). As Fig. 12 illustrates, the least precise approximation is $2|\ldots| 2$, the most accurate is $n / 2 \mid 2$ (where we introduce effective probabilities only at the lowest level), and all other variants are in between these two (in Fig. 12 this area is shaded). The approximation given by Eq. (9) is the least precise. As one can see, the quality of the best approximation, $n / 2 \mid 2$, degrades not so quickly as that of the other approximations.

We considered approximations to $\bar{K}_{16}$, which we can compute exactly, only to demonstrate the quality of different approximations. For $n \geqslant 64$ the problem of computing $\bar{K}_{n}$ with the Markov chains method is intractable, so we have to use approximations. As we have shown, the best approximation is obtained by nesting exact values of $\bar{K}_{n^{\prime}}$ with as large $n^{\prime}$ as possible. Moreover, inner $n^{\prime}$ should not exceed outer ones. The best available approximation to $\bar{K}_{1024}$ reads as

$$
\bar{K}_{1024}(p, a) \approx \bar{K}_{32}\left(\frac{1}{\bar{K}_{32}(p, a)}, a\right) .
$$

The inverse, $1 / \bar{K}_{1024}(p, a)$, gives us the best available approximation to the distribution rate. Generally, increasingly better lower bounds on the (raw) repeater rates can be obtained by our method. Depending on $L_{0}$, which typically is $10-100 \mathrm{~km}, n=1024$ segments cover the distance $L=n L_{0}>10000 \mathrm{~km}$. Using free-space satellite links to bridge these repeaters, it is probably unnecessary to use more than 1024 segments, and thus we will never need more than two nesting levels in the approximations of this form.

\section{CONCLUSIONS}

In summary, in addition to satellite-based longdistance quantum communication links, fiber-based quantum repeaters are necessary for creating reliable large-scale quantum communication networks. Memorybased quantum repeaters are starting to be experimentally realized. Up to now knowledge about the random waiting time of a general quantum repeater has been incomplete and imprecise. Here we completely solve the problem of its probability distribution function and demonstrate that, contrary to common belief, the waiting time cannot be accurately characterized by its average value alone. Our approach is applicable to general quantum repeaters, including repeaters with finite memory effects and waiting times spent for classical communication, and it allows one to obtain the full probability distribution of the waiting time, which to a good approximation in a certain regime turns out to behave like a geometric progression. We expect that precise knowledge about the waiting-time statistics also has a significant impact on the treatment of errors in a quantum repeater such as those arising from memory dephasing. Additional probabilistic entanglement manipulations, such as entanglement distillation for suppressing the propagation of errors, can also be incorporated into a repeater rate analysis using our formalism. In the QKD context, in order to determine the secret key rate in a quantum repeater system, the raw rates must be calculated and errors must be included via the secret key fraction. In our work, putting it in this context, the focus has been on repeater raw rates which are highly non-trivial to compute in a general quantum repeater with arbitrary, probabilistic entanglement swapping. Not only did we solve a long-standing problem, eventually allowing for the assessment and creation of truly robust and reliable quantum devices, we also presented a method for compressing Markov chains that can be useful for other applications as well. 
[1] S.-J. Yang, X.-J. Wang, X.-H. Bao, and J.-W. Pan, Nature Photonics 10, 381 (2016).

[2] P. Xu, H.-L. Yong, L.-K. Chen, C. Liu, T. Xiang, X.-C. Yao, H. Lu, Z.-D. Li, N.-L. Liu, L. Li, T. Yang, C.-Z. Peng, B. Zhao, Y.-A. Chen, and J.-W. Pan, Phys. Rev. Lett. 119, 170502 (2017).

[3] L.-K. Chen, H.-L. Yong, P. Xu, X.-C. Yao, T. Xiang, Z.-D. Li, C. Liu, H. Lu, N.-L. Liu, L. Li, T. Yang, C.Z. Peng, B. Zhao, Y.-A. Chen, and J.-W. Pan, Nature Photonics 11, 695 (2017).

[4] G. Brennen, E. Giacobino, and C. Simon, New Journal of Physics 17, 050201 (2015).

[5] Y.-F. Pu, N. Jiang, W. Chang, H.-X. Yang, and C. L. . L.-M. Duan, Nature Communications 8, 15359 (2017).

[6] M. Rančić, M. P. Hedges, R. L. Ahlefeldt, and M. J. Sellars, Nature Photonics 14, 50 (2018).

[7] P. Vernaz-Gris, K. Huang, M. Cao, A. S. Sheremet, and J. Laurat, Nature Communications 9, 363 (2018).

[8] Y.-F. Hsiao, P.-J. Tsai, H.-S. Chen, S.-X. Lin, C.-C. Hung, C.-H. Lee, Y.-H. Chen, Y.-F. Chen, I. A. Yu, and Y.-C. Chen, Phys. Rev. Lett. 120, 183602 (2018).

[9] B. Jing, X.-J. Wang, Y. Yu, P.-F. Sun, Y. Jiang, S.J. Yang, W.-H. Jiang, X.-Y. Luo, J. Zhang, X. Jiang, X.-H. Bao, and J.-W. Pan, "Hybrid entanglement of three quantum memories with three photons," (2018), arXiv:1808.05393.

[10] N. Sangouard, C. Simon, H. de Riedmatten, and N. Gisin, Rev. Mod. Phys. 83, 33 (2011).

[11] For earlier treatments of quantum repeaters and distributed quantum computation using the formalism of Markov chains, see 42, and 43, respectively.

[12] M. Takeoka, S. Guha, and M. M. Wilde, Nature Communications 5, 5235 (2014).

[13] S. Pirandola, R. Laurenza, C. Ottaviani, and L. Banchi, Nature Communications 8, 15043 (2017).

[14] A brief chronological summary of the different contributions to the secret-key agreement capacity and the corresponding bounds can be found in Ref. 44 .

[15] H.-J. Briegel, W. Dür, J. I. Cirac, and P. Zoller, Phys. Rev. Lett. 81, 5932 (1998); for recent alternative approaches that do not require quantum memories and are entirely based on quantum error correction codes (so-called third-generation repeaters), see S. Muralidharan, L. Li, J. Kim, N. Lütkenhaus, M. D. Lukin, and L. Jiang, Scientific Reports 6, 20463 (2016); for yet another scheme that is based upon a deterministic method of entanglement purification, see M. Zwerger, A. Pirker, V. Dunjko, H. J. Briegel, and W. Dür, Phys. Rev. Lett. 120, 030503 (2018).

[16] D. Luong, L. Jiang, J. Kim, and N. Lütkenhaus, Applied Physics B 122, 96 (2016).

[17] N. Lo Piparo, M. Razavi, and W. J. Munro, Phys. Rev. A 96, 052313 (2017).

[18] F. Rozpedek, K. Goodenough, J. Ribeiro, N. Kalb, V. C. Vivoli, A. Reiserer, R. Hanson, S. Wehner, and D. Elkouss, Quantum Science and Technology 3, 034002 (2018).

[19] S. Abruzzo, H. Kampermann, and D. Bruß, Phys. Rev. A 89, 012303 (2014).

[20] In R. Schwonnek, Dynamische Modellierung von Quantenrepeater-Prozessen, Master's thesis, Institut für Theoretische Physik der Leibniz Universität Hannover
(2013), methods to calculate rates in memory-based quantum repeaters have been presented with a particular focus on imperfect quantum memories.

[21] L.-M. Duan, M. D. Lukin, J. I. Cirac, and P. Zoller, Nature 414, 413 (2001).

[22] J. Calsamiglia and N. Lütkenhaus, Applied Physics B 72, 67 (2001)

[23] J. Borregaard, P. Kómár, E. M. Kessler, M. D. Lukin, and A. S. Sørensen, Phys. Rev. A 92, 012307 (2015).

[24] S. Abruzzo, S. Bratzik, N. K. Bernardes, H. Kampermann, P. van Loock, and D. Bruß, Phys. Rev. A 87, 052315 (2013).

[25] S. Bratzik, S. Abruzzo, H. Kampermann, and D. Bruß, Phys. Rev. A 87, 062335 (2013).

[26] M. Uphoff, M. Brekenfeld, G. Rempe, and S. Ritter, Applied Physics B 122, 46 (2016).

[27] W. P. Grice, Phys. Rev. A 84, 042331 (2011).

[28] H. A. Zaidi and P. van Loock, Phys. Rev. Lett. 110, 260501 (2013).

[29] F. Ewert and P. van Loock, Phys. Rev. Lett. 113, 140403 (2014).

[30] N. K. Bernardes, L. Praxmeyer, and P. van Loock, Phys. Rev. A 83, 012323 (2011).

[31] In order to calculate a secret key rate in quantum repeater-based communication, besides the raw rate, inclusion of errors requires determining the quantum bit error rate. Vinay and Kok [45], building upon an earlier version of our work, explicitly showed already for a particular repeater protocol how to incorporate errors into the Markov-chain approach.

[32] O. A. Collins, S. D. Jenkins, A. Kuzmich, and T. A. B. Kennedy, Phys. Rev. Lett. 98, 060502 (2007).

[33] L. Praxmeyer, "Reposition time in probabilistic imperfect memories," (2013), arXiv:1309.3407.

[34] F. K. Asadi, N. Lauk, S. Wein, N. Sinclair, C. O'Brien, and C. Simon, Quantum 2, 93 (2018).

[35] J. G. Kemeny and J. L. Snell, Finite Markov chains (Springer-Verlag, 1976).

[36] https://www.numpy.org/.

[37] P. Bremaud, Markov chains. Gibbs fields, Monte Carlo simulation, and queues (Springer-Verlag, 1999).

[38] In an earlier version of our work, we had already included this alternative approach. Later Vinay and Kok 45] put a greater emphasis on such methods and also demonstrated the usefulness of (probability) generating functions for analyzing the rates and the statistics of a quantum repeater.

[39] In an earlier version of our work, we had already included the lumbability method as a means to render the Markovchain-based rate analysis more efficient. Vinay and Kok 45. employ and describe similar methods.

[40] https://oeis.org/A007501.

[41] S. Santra, L. Jiang, and V. S. Malinovsky, Quantum Science and Technology 4, 025010 (2019).

[42] M. Razavi, K. Thompson, H. Farmanbar, M. Piani, and N. Lütkenhaus, Proc.SPIE 7236, 723603 (2009).

[43] L. Jiang, J. M. Taylor, A. S. Sørensen, and M. D. Lukin, Phys. Rev. A 76, 062323 (2007).

[44] M. Pant, H. Krovi, D. Towsley, L. Tassiulas, L. Jiang, P. Basu, D. Englund, and S. Guha, npj Quantum Information 5, 25 (2019). 
[45] S. E. Vinay and P. Kok, Phys. Rev. A 99, 042313 (2019). 